\title{
Formation Mechanism of Maghemite Nanoflowers Synthesized by a Polyol-Mediated Process
}

Gavilán, Helena; Sánchez, Elena H.; Brollo, María E. F.; Asín, Laura; Moerner, Kimmie Katrine; Frandsen, Cathrine; Lázaro, Francisco J.; Serna, Carlos J.; Veintemillas-Verdaguer, Sabino; Morales, M. Puerto Total number of authors:

11

Published in:

ACS Omega

Link to article, DOI:

10.1021/acsomega.7b00975

Publication date:

2017

Document Version

Publisher's PDF, also known as Version of record

Link back to DTU Orbit

Citation (APA):

Gavilán, H., Sánchez, E. H., Brollo, M. E. F., Asín, L., Moerner, K. K., Frandsen, C., Lázaro, F. J., Serna, C. J., Veintemillas-Verdaguer, S., Morales, M. P., \& Gutiérrez, L. (2017). Formation Mechanism of Maghemite Nanoflowers Synthesized by a Polyol-Mediated Process. ACS Omega, 2(10), 7172-7184.

https://doi.org/10.1021/acsomega.7b00975

\section{General rights}

Copyright and moral rights for the publications made accessible in the public portal are retained by the authors and/or other copyright owners and it is a condition of accessing publications that users recognise and abide by the legal requirements associated with these rights.

- Users may download and print one copy of any publication from the public portal for the purpose of private study or research.

- You may not further distribute the material or use it for any profit-making activity or commercial gain

- You may freely distribute the URL identifying the publication in the public portal 


\title{
Formation Mechanism of Maghemite Nanoflowers Synthesized by a Polyol-Mediated Process
}

\author{
Helena Gavilán, ${ }^{* \dagger}$ Elena H. Sánchez, ${ }^{\dagger}$ María E. F. Brollo, ${ }^{\dagger}$ Laura Asín, ${ }^{\ddagger}$ Kimmie K. Moerner, ${ }^{\S}$
}

Cathrine Frandsen, ${ }^{\S}$ Francisco J. Lázaro, ${ }^{\|}$Carlos J. Serna, ${ }^{\dagger}$ Sabino Veintemillas-Verdaguer, ${ }^{\dagger}$ M. Puerto Morales, ${ }^{*}{ }^{\dagger} \odot$ and Lucía Gutiérrez $\left.{ }^{*, \perp}{ }^{(}\right)$

\begin{abstract}
${ }^{\dagger}$ Materials Science Factory, Institute of Materials Science of Madrid/CSIC (ICMMCSIC), Sor Juana Inés de la Cruz 3, 28049 Madrid, Spain

${ }^{\ddagger}$ Institute of Materials Science of Aragón, Universidad de Zaragoza, CSIC and CIBER-BBN, Campus Río Ebro, Edificio I+D, Mariano Esquillor Gómez, 50018 Zaragoza, Spain

${ }^{\S}$ Department of Physics, Technical University of Denmark, Fysikvej, Building 307, 2800 Kgs. Lyngby, Denmark

"Departamento de Ciencia y Tecnología de Materiales y Fluidos, Universidad de Zaragoza, María de Luna 3, 50018 Zaragoza, Spain

${ }^{\perp}$ Department of Analytical Chemistry, Universidad de Zaragoza and CIBER-BBN, Fundación Instituto Universitario de Nanociencia de Aragón (INA), Edificio I+D, Mariano Esquillor Gómez, 50018 Zaragoza, Spain
\end{abstract}

\section{Supporting Information}

ABSTRACT: Magnetic nanoparticles are being developed as structural and functional materials for use in diverse areas, including biomedical applications. Here, we report the synthesis of maghemite $\left(\gamma-\mathrm{Fe}_{2} \mathrm{O}_{3}\right)$ nanoparticles with distinct morphologies: single-core and multicore, including hollow spheres and nanoflowers, prepared by the polyol process. We have used sodium acetate to control the nucleation and assembly process to obtain the different particle morphologies. Moreover, from samples obtained at different time steps during the synthesis, we have elucidated the formation mechanism of the nanoflowers: the initial phases of the reaction present a lepidocrocite $(\gamma-\mathrm{FeOOH})$ structure, which suffers a fast dehydroxylation, transforming to an intermediate
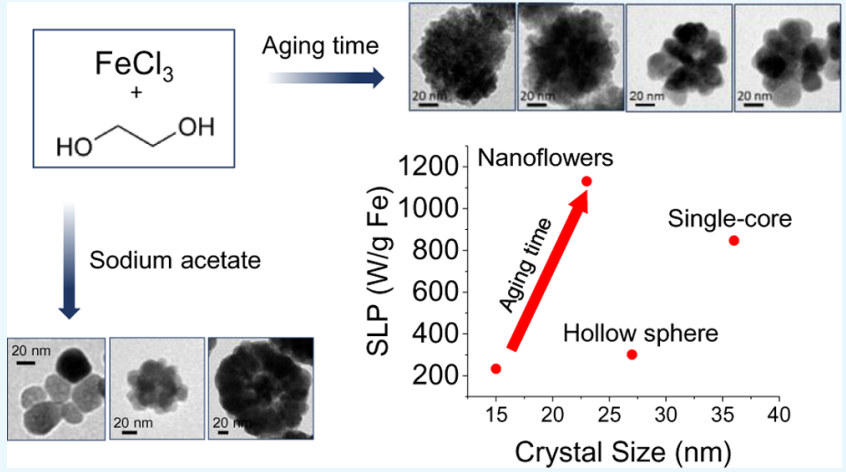
"undescribed" phase, possibly a partly dehydroxylated lepidocrocite, which after some incubation time evolves to maghemite nanoflowers. Once the nanoflowers have been formed, a crystallization process takes place, where the $\gamma$-Fe $\mathrm{O}_{3}$ crystallites within the nanoflowers grow in size (from $\sim 11$ to $23 \mathrm{~nm}$ ), but the particle size of the flower remains essentially unchanged $(\sim 60 \mathrm{~nm}$ ). Samples with different morphologies were coated with citric acid and their heating capacity in an alternating magnetic field was evaluated. We observe that nanoflowers with large cores $(23 \mathrm{~nm}$, controlled by annealing) densely packed (tuned by low NaAc concentration) offer 5 times enhanced heating capacity compared to that of the nanoflowers with smaller core sizes (15 $\mathrm{nm}$ ), 4 times enhanced heating effect compared to that of the hollow spheres, and 1.5 times enhanced heating effect compared to that of single-core nanoparticles $(36 \mathrm{~nm})$ used in this work.

\section{INTRODUCTION}

Through the polyol-mediated synthesis developed by Fievet, Lagier, and Figlarz, the formation of both noble metals and metal oxide nanocrystals has been widely reported, obtaining monodispersed systems thanks to the polyol acting as the surfactant of the as-synthesized particles, reducer, and reaction media. ${ }^{2}$ The first advantage of this procedure is that it allows the synthesis of hydrophilic particles that remain stable in aqueous media ${ }^{3}$ and other polar solvents within one step, ${ }^{4}$ avoiding further coating steps required by other synthesis routes such as thermal decomposition of organometallic precursors. In addition, owing to the high dielectric constants of the polyols, ${ }^{5}$ they are able to dissolve inorganic compounds used as reactants, and due to their relatively high boiling points, great crystallinity of the particles can be achieved.

Several examples of the synthesis of metal nanoparticles (NPs) achieved by this procedure exist in the literature. Among others, functional materials, such as $\mathrm{Au}-\mathrm{Pd}$ colloidal nanoparticles for catalysis purposes ${ }^{6}$ and Co nanorods for the development of new permanent magnets, ${ }^{7}$ have been recently synthesized. Besides noble metals, the synthesis of both singlecore (SC) and multicore magnetic nanoparticles ${ }^{8}$ has been reported through polyol reduction, organizing colloidal

Received: July 11, 2017

Accepted: September 12, 2017

Published: October 26, 2017 
magnetic cores into highly regular nanoparticles with tuned properties. Of special interest are iron oxide multicore nanoparticles assembled in flower-shaped structures synthesized by polyols. ${ }^{9,10}$ In comparison with the single-core counterparts, these flower-like nanoparticles have shown enhanced longitudinal and transverse relaxivities for magnetic resonance imaging contrast generation ${ }^{11}$ and enhanced specific absorption rate (SAR) values for magnetic hyperthermia due to hysteresis heating. ${ }^{12}$ The interest in magnetite $\left(\mathrm{Fe}_{3} \mathrm{O}_{4}\right)$ and maghemite $\left(\gamma-\mathrm{Fe}_{2} \mathrm{O}_{3}\right)$ nanoparticles for biomedical applications, both in diagnosis ${ }^{13}$ and therapy, ${ }^{14,15}$ relies on their biocompatibility $^{16,11}$ and nontoxicity, ${ }^{17}$ chemical stability, and the strong response when exposed to an external magnetic field. ${ }^{18}$

The assembly process and particle and core sizes are crucial characteristics that determine the magnetic properties of the colloid and therefore its optimal use for a given application. ${ }^{19,20}$ In the present work, we have analyzed the assembly process that leads to the formation of single-core and multicore hollow and flower-like maghemite nanoparticles through the polyol process. We have also determined the experimental parameters that allow control of the core sizes within the nanoflowers (NFs). In detail, we have analyzed the role of specific experimental conditions in the synthesis, such as the presence of a precipitator (sodium acetate, $\mathrm{NaAc}$ ), an extra stabilizer (poly(vinylpyrrolidone), PVP), and the aging time. Then, the consequences of the different morphologies and core sizes on the magnetic cooperative behavior have been studied. Finally, some selected samples were coated with citric acid (CA) and the possible use of these particles for magnetic hyperthermia applications has been evaluated through specific loss power (SLP) measurements.

\section{EXPERIMENTAL SECTION}

Materials. Ferric chloride hexahydrate $\left(\mathrm{FeCl}_{3} \cdot 6 \mathrm{H}_{2} \mathrm{O}, \geq 98 \%\right.$; Sigma-Aldrich), poly(vinylpyrrolidone) (PVP40; Sigma-Aldrich), sodium acetate trihydrate $\left(\mathrm{NaAc} \cdot 3 \mathrm{H}_{2} \mathrm{O}, \geq 99 \%\right.$; SigmaAldrich), ethylene glycol (EG, $\geq 99.5 \%$; Fluka), citric acid $\left(\mathrm{C}_{6} \mathrm{H}_{8} \mathrm{O}_{7}, \geq 99.5 \%\right.$; Sigma-Aldrich), and agar (2\%; Panreac) were obtained and used without any further treatment.

Synthesis of Nanoparticles. The synthesis of iron oxide nanoparticles has been on the basis of a previous work described in the literature, ${ }^{14}$ but the experimental procedure and the concentration of $\mathrm{NaAc}$ and PVP have been varied. Typically, $2.62 \mathrm{mmol} \mathrm{FeCl}_{3} \cdot 6 \mathrm{H}_{2} \mathrm{O}$ was dissolved with ultrasound in $109 \mathrm{~mL}$ of ethylene glycol. Then, $140 \mathrm{mmol}$ PVP40 was added slowly under vigorous magnetic stirring $(>1000$ rpm) and mild heating until completely dissolved. Then, 15.8$36.5 \mathrm{mmol} \mathrm{NaAc} \cdot 3 \mathrm{H}_{2} \mathrm{O}$ was added to the solution. The mixture was sealed in a Teflon-lined autoclave $(125 \mathrm{~mL})$ and maintained at $200{ }^{\circ} \mathrm{C}$ for $0.5-48 \mathrm{~h}$ for solvothermal crystallization, followed by cooling inside an oven. The precipitated solid product was washed with ethanol and distilled water through centrifugation several times.

Surface Coating. For citric acid coating, a standard procedure was used. ${ }^{8,21}$ First, sample volume equivalent to 20 $\mathrm{mg}$ of $\mathrm{Fe}$ was adjusted to $\mathrm{pH} 2$ and then dispersed in $13 \mathrm{~mL}$ of a solution of $0.1 \mathrm{M}$ citric acid. Afterward, the mixture was heated at $80{ }^{\circ} \mathrm{C}$ for $30 \mathrm{~min}$. The solution was centrifuged and washed with distilled water. Finally, the $\mathrm{pH}$ was adjusted first to 11 with $1 \mathrm{M} \mathrm{KOH}$ and then to 7 with $0.01 \mathrm{M} \mathrm{HNO}_{3}$.

Characterization. The particle sizes and morphologies were determined by transmission electron microscopy (TEM) with a JEM1010 microscope (JEOL, Peabody) operating at 100
$\mathrm{kV}$. Samples were prepared by placing a drop of the uncoated particles suspended in water onto a carbon-coated copper grid and allowing it to dry at room temperature (RT). The size distributions were determined by manual measurement of more than 100 particles using the public domain software ImageJ.

The crystal structure was identified by X-ray diffraction (XRD) performed on freeze-dried powders in an X'pert PRO diffractometer from Panalytical with a Johansson monochromator and using $\mathrm{Cu} \mathrm{K} \alpha$ radiation $(\lambda=1.5406 \AA)$. The patterns were collected within a $2 \theta$ range of $10-90^{\circ}$ at a scan rate of $0.04^{\circ} / \mathrm{min}$. The crystallite size, $d_{\mathrm{XRD}}$, of the maghemite samples was determined by Scherrer's formula, using the (311) diffraction line. The presence of the coating and the phase evolution was also confirmed by Fourier transform infrared (FTIR) spectroscopy in the range of $4000-250 \mathrm{~cm}^{-1}$ by use of a Bruker IFS 66VS. The samples for FTIR were prepared by diluting the dried powder in $\mathrm{KBr}$ at $2 \%$ by weight and pressing it into pellets.

Colloidal properties were studied in a Zetasizer Nano $S$, from Malvern Instruments (U.K.). The hydrodynamic size, $D_{\mathrm{h}}$, was determined by dynamic light scattering (DLS), and the $\zeta$ potential was measured as a function of $\mathrm{pH}$ at $25{ }^{\circ} \mathrm{C}$ using $\mathrm{HNO}_{3}$ and $\mathrm{KOH}$ to change the $\mathrm{pH}$ of the suspensions. $D_{\mathrm{h}}$ is given as the intensity-weighted and number-weighted mean value to compare to the TEM mean values.

For the magnetic characterization, the samples were measured in powder form after drying in an inox-coated oven at $50{ }^{\circ} \mathrm{C}$. After accurately weighing a mass of $\approx 10 \mathrm{mg}$, the powder was filled into polycarbonate capsules and immobilized with cotton wool. Hysteresis loops were measured in a vibrating sample magnetometer (MLVSM9, MagLab 9T; Oxford Instruments, U.K.) at 5 and $290 \mathrm{~K}$ in fields up to $4000 \mathrm{kA} / \mathrm{m}$ at a field change rate of $240 \mathrm{kA} / \mathrm{m}$ min after saturating the sample in a $4000 \mathrm{kA} / \mathrm{m}$ field. Alternating current (AC) susceptibility measurements were performed in a Quantum Design MPMS$\mathrm{XL}$ superconducting quantum interference device magnetometer using the same capsules described above. Data was collected in the temperature range between 2 and $300 \mathrm{~K}$ using magnetic field amplitude of $0.41 \mathrm{mT}$ and frequency of $11 \mathrm{~Hz}$. Additional measurements at 0.11 and $110 \mathrm{~Hz}$ were performed in selected samples.

To further elucidate the composition of the samples and their magnetic properties, ${ }^{57} \mathrm{Fe}$ Mössbauer spectra were obtained in transmission geometry on freeze-dried samples at 18,80 , and $295 \mathrm{~K}$, using a constant acceleration spectrometer from WissEl $\mathrm{GmbH}$ and a flux-closed helium refrigerator from APD Cryogenics. Spectra have been fit using Lorentzian-shaped lines. Isomer shifts are given relative to that of $\alpha$-Fe.

The evaluation of heat generation was determined by a commercial AC-field applicator (DM100; Nanoscale Biomagnetics, Spain). Samples, either in water or in a viscous media (agar $2 \% \mathrm{w} / \mathrm{v}$ ), were placed in a closed container centered in the inductive coil and the AC-field was applied for $5 \mathrm{~min}$. The applied field amplitude was $H=24 \mathrm{kA} / \mathrm{m}$, and the applied frequencies were 419,542 , and $710 \mathrm{kHz}$. The temperature of the sample was recorded by an optic fiber sensor incorporated in the equipment.

\section{RESULTS AND DISCUSSION}

In this work, we have made use of the conventional polyolmediated route, utilizing an iron(III) salt in EG, followed by a solvothermal reaction in the presence of PVP and NaAc, which yields multicore magnetic NPs, also called magnetic nanocrystal 
clusters. ${ }^{22}$ Changes introduced in the experimental conditions (NaAc concentration and heating time) have successfully allowed us to modulate the NPs' size and the size and configuration of the magnetic cores.

For that purpose, some relevant parameters were initially fixed to assure the formation of monodispersed colloids with particle sizes below $200 \mathrm{~nm}$, which is a key issue to guarantee colloidal stability and to make this suspension potentially more suitable for biomedical applications. The first parameter is the choice of polyol and especially its length, which has been shown to influence the size and the assembly of the magnetic cores. ${ }^{23}$ In this work, ethylene glycol (EG) has been selected, as it is a short polyol that allows clustering such that multicore particles can be obtained. Polyols with longer chains generally lead to single-core nanoparticles ${ }^{4,24}$ (depending on the rest of the reagents present in the reaction vessel). The second parameter is the addition of PVP, which has been shown to provide extra stability because it acts as a capping agent. The third parameter is the iron/PVP concentration, which determines the particle size. Polyol-based synthesis of iron(III) salts in an autoclave using polyols of short chain and stabilizers such as PVP or poly(ethylene glycol) usually leads to uniform particles of around $300 \mathrm{~nm} .{ }^{14,25}$ The use of a base, namely, sodium hydroxide ${ }^{23}$ or urea, ${ }^{26,27}$ has accounted for a further reduction of size. In this work, the iron/PVP concentration was fixed to achieve particles smaller than $200 \mathrm{~nm}$.

This section is divided in three parts. First, the role of $\mathrm{NaAc}$ in the growth and assembly process is evaluated. Then, the formation mechanism of the nanoflowers is analyzed. Lastly, four samples with different morphologies (different assembly configurations) and crystallite sizes are selected and functionalized with citric acid such that they form stable magnetic colloids and their different potential for magnetic hyperthermia treatments are evaluated.

Role of Sodium Acetate in the Assembly Process. The amount of $\mathrm{NaAc} \cdot 3 \mathrm{H}_{2} \mathrm{O}$ was varied from 15.8 to $36.5 \mathrm{mmol}$ (Table 1) while maintaining the rest of the reactants and conditions. Figure 1 shows the normalized log-normal size distributions of the samples, obtained by manual measurement
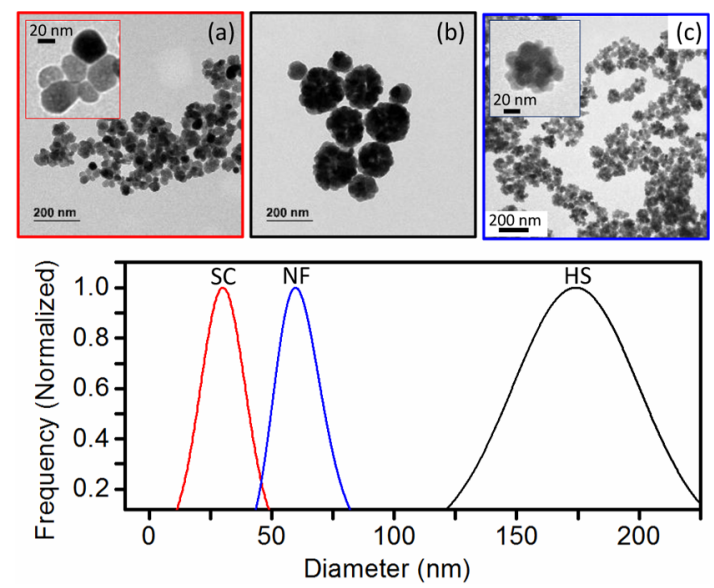

Figure 1. TEM images of the different structures obtained when varying the NaAc amount: (a) single-core (SC) particles, $36.5 \mathrm{mmol}$; (b) hollow spheres (HS), $26.2 \mathrm{mmol}$; and (c) nanoflowers (NF), 15.8 mmol. Below are the normalized log-normal TEM size distributions of the samples. Please note that in solution, the hydrodynamic diameters of the as-synthesized samples SC, HS, and NF were 900, 700, and 300 $\mathrm{nm}$, respectively. of the particle diameter through ImageJ software and further data fitting using Origin software. In all cases, monodispersed systems with narrow size distributions were achieved thanks to the presence of PVP (the standard deviation was below 25\%). Figure $S 1$ shows the particles obtained in the absence of PVP with irregular morphology (mean size of $200 \mathrm{~nm}$ and standard deviation of $48 \%$ ). We found that the variation of $\mathrm{NaAc}$ concentration leads to nanoparticles with distinctly different structures (Figure 1). The highest concentration of NaAc (36.5 $\mathrm{mmol}$ ) produced single-core nanoparticles of $35 \mathrm{~nm}$ (sample SC, Figure 1a). These particles appear under the TEM as randomly distributed and somewhat aggregated (possibly due to the drying process on the TEM grid), but no specific assembly of the single-core particles was observed.

Table 1. Sodium Acetate Optimization ${ }^{a}$

$\begin{array}{ccccc}\text { sample } & {[\mathrm{NaAc}](\mathrm{mmol})} & \text { time }(\mathrm{h}) & d_{\text {TEM }}(\mathrm{nm}) & d_{\text {XRD }}(\mathrm{nm}) \\ \text { SC } & 36.5 & 16 & 35 \pm 8 & 36.2 \pm 0.1 \\ \text { HS } & 26.2 & 16 & 170 \pm 30 & 27.4 \pm 0.2 \\ \text { NF } & 15.8 & 16 & 63 \pm 13 & 22.9 \pm 0.2\end{array}$

${ }^{a}$ Experimental conditions, TEM size distribution, and XRD size of maghemite nanoparticles with different morphologies, including single-core (SC), hollow spheres (HS), and nanoflowers (NF).

However, when decreasing the $\mathrm{NaAc}$ concentration to 26.2 mmol, spherical hollow multicore nanoparticles about $170 \mathrm{~nm}$ in diameter were formed (sample HS, Figure 1b). TEM images reveals lower material density in the inner part, which is attributed to the hollow structure. ${ }^{14}$ Figure S2 further supports the existence of voids within the spheres (again due to contrast between the darker edges of the particle and the brighter centers). The lowest amount of NaAc $(15.8 \mathrm{mmol})$ yielded multicore particles with a flower-like structure (sample NF, Figure 1c).

In this case, the cores $(<20 \mathrm{~nm})$ are densely packed, forming a nanoflower particle with a characteristic size of $\sim 60 \mathrm{~nm}$.

In solution, as indicated in the figure caption of Figure 1, the intensity-weighted hydrodynamic diameters of the as-synthesized samples SC, HS, and NF were 900, 700, and $300 \mathrm{~nm}$, respectively, well above the TEM size. This is due to the presence of polyol and PVP, which was detected through FTIR spectroscopy (Figure S6a); the low surface charge of the assynthesized samples (Figure 9b, dashed line); and as a consequence of their magnetic character.

Figure 2 shows the XRD patterns of samples SC, HS, and $\mathrm{NF}$, which correspond to a ferrite spinel structure attributed to $\gamma-\mathrm{Fe}_{2} \mathrm{O}_{3}$ (JCPDS \#110614). An increase in the broadening of the Bragg peaks, which indicates a decreasing crystallite size, is observed from SC to HS and again to NF. The crystal sizes $d_{\mathrm{XRD}}$ (Table 1) determined by Scherrer's equation agree well with $d_{\mathrm{TEM}}$ in the case of single-core (SC) particles $\left(d_{\mathrm{XRD}}=36\right.$ $\mathrm{nm})$, but in the case of NF and HS, $d_{\mathrm{XRD}}$ is significantly smaller than $d_{\text {TEM }}$. This discrepancy reflects the multicore nature of these samples. ${ }^{19}$ From TEM and XRD, it is clear that decreasing the amount of NaAc leads to more densely packed assemblies with smaller core sizes.

Figure 3 shows the field dependence of magnetization of the three different particle morphologies: SC, HS, and NF, at 290 and $5 \mathrm{~K}$. The insets in Figure 3 show the hysteresis loops in the low-field regime, and Table 2 summarizes the saturation magnetization $\left(M_{s}\right)$, initial mass susceptibility at low fields $\left(\chi_{0}\right)$, squareness $\left(M_{\mathrm{r}} / M_{\mathrm{s}}\right.$ ratio $)$, and coercivity $\left(H_{\mathrm{c}}\right)$. 


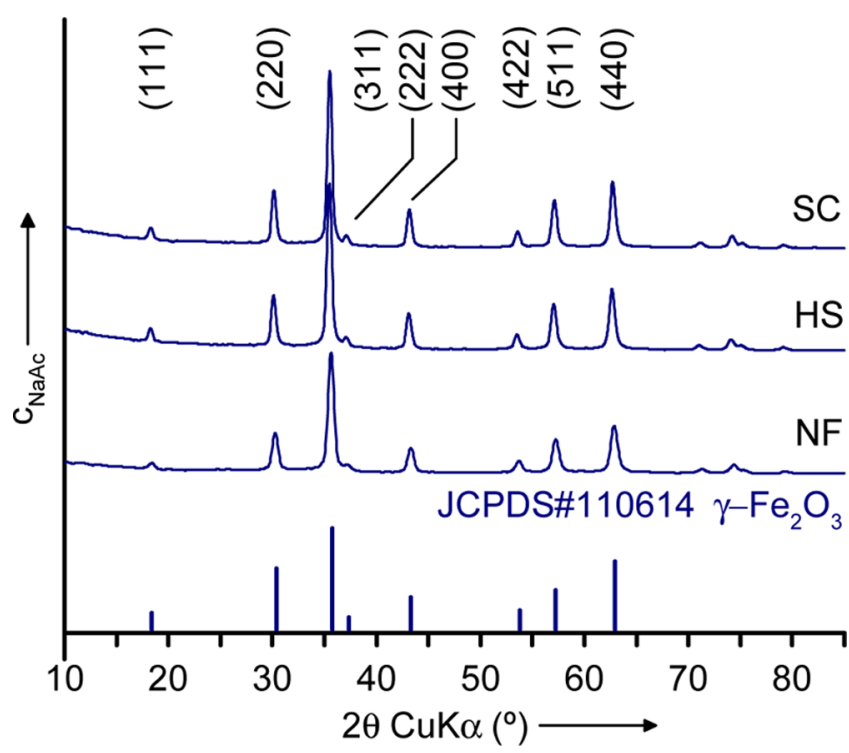

Figure 2. X-ray diffractogram of single-core (SC) particles, hollow spheres (HS), and nanoflowers (NF).

Please note that $M_{s}$ obtained in this work is formally the magnetic moment per unit mass (This is generally termed as the specific magnetization, $\sigma$.) and the $\chi_{0}$ is calculated through the numerical field derivative of $M$. The samples have $M_{\mathrm{s}}$ values between 80 and $90 \mathrm{~A} \mathrm{~m}^{2} / \mathrm{kg}$ at room temperature and go up to $100 \mathrm{~A} \mathrm{~m}^{2} / \mathrm{kg}$ at $5 \mathrm{~K}$. As with $\mathrm{XRD}$, saturation magnetization is larger for sample SC, than for samples $H S$ and NF, i.e. $M_{S}$ increases with core size. Enlargement of the low-field sections of the curves are shown in the insets to get a more accurate view of hysteresis effects. Samples SC and HS show larger coercivity values of 4.5 and $4 \mathrm{kA} / \mathrm{m}$, respectively, at $290 \mathrm{~K}$, in contrast to those of sample NF that shows nearly zero coercivity and remanence at $290 \mathrm{~K}$. All samples, regardless of their structure, have coercivity values between 14 and $17 \mathrm{kA} / \mathrm{m}$ at low temperature, which are of the same order of magnitude as the coercivity reported for magnetite-maghemite nanoparticles with only magnetocrystalline anisotropy. ${ }^{28}$ Also, the initial mass susceptibility $\left(1.31-1.70 \mathrm{~m}^{3} / \mathrm{kg}\right)$ at room temperature and the low squareness ratio $(0.21-0.23)$ at $5 \mathrm{~K}$ are comparable for the three materials.

The above results show the fundamental role of $\mathrm{NaAc}$ in this synthesis route because it directly defines the core size and morphology of the samples and in turn leads to nanoparticles with different magnetic behaviors. The reason underlies in the chemical process taking place. It is known that both $\mathrm{NaAc}$ and water need to be present in the reaction to modify the reduction potential of the polyol that otherwise is not able to reduce the iron reactants. ${ }^{29}$ Moreover, its concentration defines the self-assembly process. Thus, for the highest acetate concentration $(0.3 \mathrm{M})$, massive nucleation and uniform growth by diffusion take place, resulting in single-core particles. When the amount of acetate is reduced $(0.25 \mathrm{M})$, the initial nuclei seem to grow by partial aggregation in hollow spheres.

Other studies have reported the formation of similar hollow spheres by dissolution of inner small cores and growth of the larger ones on the surface by the Ostwald ripening process. ${ }^{14}$ Further reduction in acetate $(<0.2 \mathrm{M})$ leads to smaller nuclei that strongly aggregate to produce the final flower-like particles. In absence of $\mathrm{NaAc}$, no precipitation occurred, as previously reported. . $^{30}$

Formation Mechanism of Multicore Nanoflowers. To study the formation mechanism and magnetic properties of multicore nanoflowers, the $\mathrm{NaAc}$ concentration $(15.8 \mathrm{mmol})$ was fixed and the heating time was varied from 0.5 to $48 \mathrm{~h}$. All NF samples are named according to their heating time (measured in hours). For this series of samples, NF-16 is equivalent to sample NF studied above. First, electron microscopy was used to study the evolution of nanoflower formation. Figure 4 shows the as-synthesized product at the different reaction times. At short reaction times ( $<30 \mathrm{~min})$, a reddish colloidal suspension is observed, consisting of primary nuclei of 2-3 nm, unstable under the TEM electron beam. After $1.8 \mathrm{~h}$, there is a reddish-brown precipitate, which corresponds with nuclei that rarely approach and form aggregates that are poorly defined (as distinctly observed in the inset image of Figure 4). After $2 \mathrm{~h}$ of aging time, the primary nuclei seem to be transformed into a material with a laminar habit (sheets that have lower contrast than that of the nanoflowers $)$ and the first nanoflowers $(60 \mathrm{~nm}$ in size) are observed. The laminar phase disappears after $4 \mathrm{~h}$. The nanoflower size is preserved $\left(d_{\mathrm{TEM}} \approx 60 \mathrm{~nm}\right)$ between 2 and $48 \mathrm{~h}$, see Table 3 , but a clear increase of the core size is observed over time. Thus, TEM images reveal two different stages in the mechanism of flower formation. The first one is an initial stage $(<2 \mathrm{~h}$ of heating time) where several intermediate phases may coexist. The second one $(2-48 \mathrm{~h})$ comprises the growth of the cores within a nanoflower, which does not change in its total size $(\approx 60 \mathrm{~nm})$. Figure S3 shows the histograms obtained from TEM measurements of the nano-
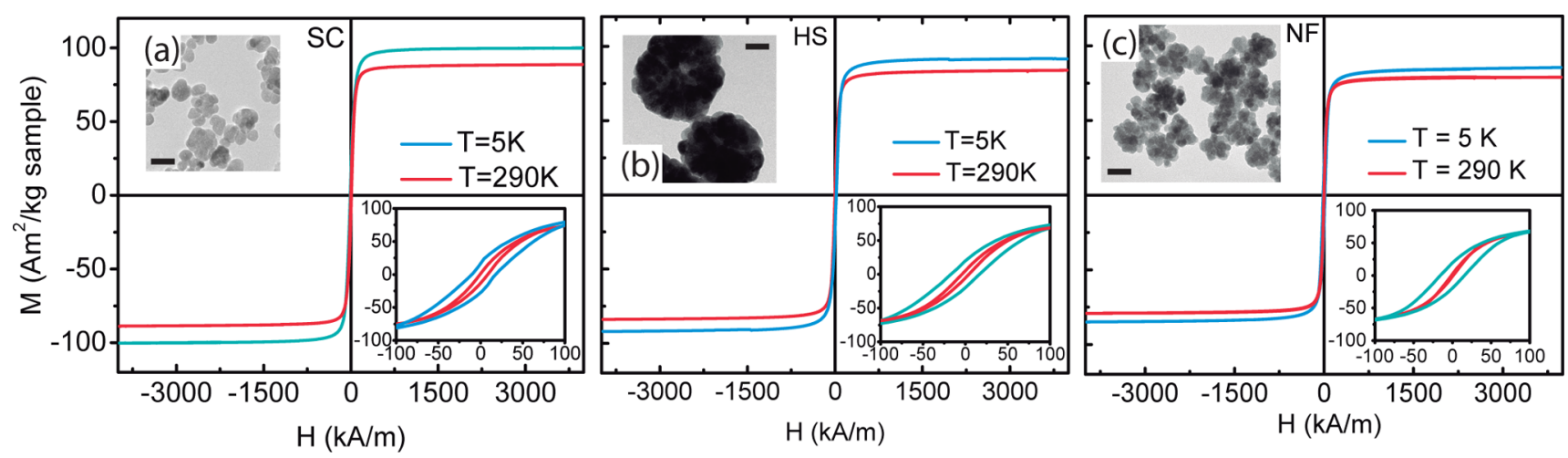

Figure 3. Magnetization curves for single-core (SC) particles (a), hollow spheres (HS) (b), and nanoflowers (NF) (c) at 5 and $290 \mathrm{~K}$. Inset TEM images of each sample whose scale bar represents $50 \mathrm{~nm}$ and low-field regime hysteresis loops. 
Table 2. Hysteresis Parameters at Room Temperature and $5 \mathrm{~K}$ for Samples SC, HS, and NF

\begin{tabular}{|c|c|c|c|c|c|c|}
\hline \multirow[b]{2}{*}{ sample } & \multicolumn{3}{|c|}{ RT } & \multicolumn{3}{|c|}{$5 \mathrm{~K}$} \\
\hline & $M_{\mathrm{s}}\left(\mathrm{A} \mathrm{m}^{2} / \mathrm{kg}\right)$ & $H_{\mathrm{c}}(\mathrm{kA} / \mathrm{m})$ & $\chi_{0}\left(\mathrm{~m}^{3} / \mathrm{kg}\right)$ & $M_{\mathrm{s}}\left(\mathrm{A} \mathrm{\textrm {m } ^ { 2 }} / \mathrm{kg}\right.$ sample $)$ & $M_{\mathrm{r}} / M_{\mathrm{s}}$ & $\overline{H_{\mathrm{c}}(\mathrm{kA} / \mathrm{m})}$ \\
\hline $\mathrm{SC}$ & $90.2 \pm 0.1$ & 4 & $1.70 \pm 0.02$ & $100.3 \pm 0.1$ & $0.23 \pm 0.1$ & 14 \\
\hline HS & $84.3 \pm 0.1$ & 4 & $1.31 \pm 0.01$ & $92.5 \pm 0.1$ & $0.21 \pm 0.1$ & 17 \\
\hline NF & $79.7 \pm 0.1$ & 1 & $1.60 \pm 0.02$ & $85.5 \pm 0.1$ & $0.22 \pm 0.1$ & 16 \\
\hline
\end{tabular}

Initial stages of the flower formation ( $<2 \mathrm{~h}$ of heating time)

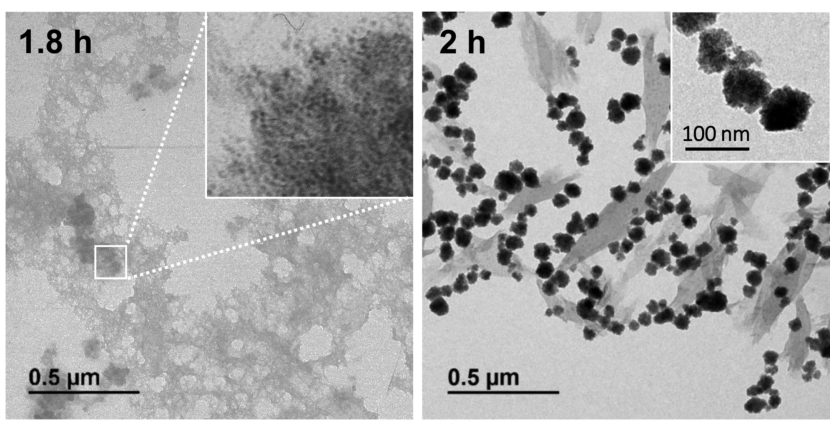

Aging process: crystal growth (2-48 h)
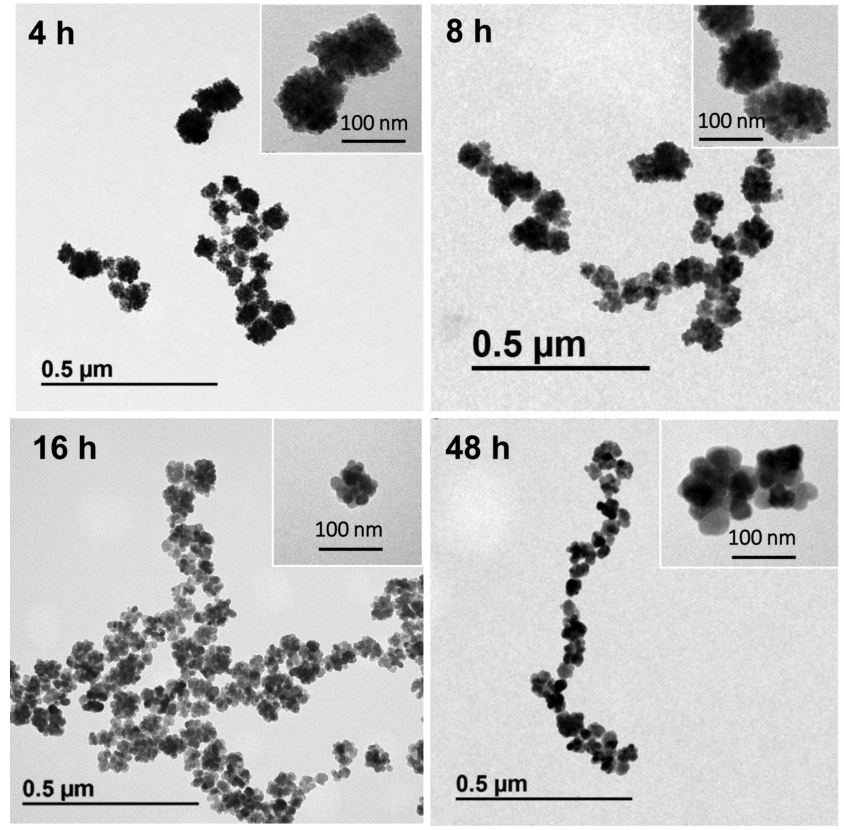

Figure 4. TEM images of NF samples prepared with $15.8 \mathrm{mmol} \mathrm{NaAc}$ at different reaction times: $1.8,2,4,8,16$, and $48 \mathrm{~h}$. Insets show a detailed image of a single nanoflower.

Table 3. TEM and XRD Sizes during the Formation of Nanoflower Particles ${ }^{a}$

$\begin{array}{lccc}\text { sample } & \text { time }(\mathrm{h}) & d_{\text {TEM }}(\mathrm{nm}) & d_{\text {XRD }}(\mathrm{nm}) \\ \text { NF-0.5 } & 0.5 & & \\ \text { NF-1 } & 1 & & \\ \text { NF-1.8 } & 1.8 & & \\ \text { NF-2 } & 2 & 60 \pm 11 & 11.3 \pm 0.3 \\ \text { NF-4 } & 4 & 56 \pm 13 & 14.8 \pm 0.3 \\ \text { NF-8 } & 8 & 61 \pm 16 & 19.2 \pm 0.2 \\ \text { NF-16 } & 16 & 63 \pm 13 & 22.9 \pm 0.2 \\ \text { NF-48 } & 48 & 58 \pm 11 & 23.7 \pm 0.2\end{array}$

${ }^{a}$ For all of these syntheses, [NaAc] was kept at $15.8 \mathrm{mmol}$.

flower sizes. This data was fitted to a log-normal size distribution (Figure S4), and it seems that the size distribution gets narrower after longer periods of heating time. This is in good agreement with a two-stage mechanism of nanoparticle formation by self-assembly of diffusing aggregating nanocrystalline subunits, which are in turn formed by burst nucleation in a supersaturated solution, followed by coarsening. ${ }^{31}$ A detailed analysis of both stages is provided in the following sections.

Initial Stages of the Flower Formation $(<2$ h). To identify the different phases that appear during these initial stages, the composition of samples NF-0.5-2 were evaluated by $\mathrm{XRD}$ (Table 3). For all of these syntheses, [NaAc] was kept at $15.8 \mathrm{mmol}$. Figure 5a shows the X-ray diffraction pattern of the NF samples. At $t=0.5 \mathrm{~h}$, the pattern clearly matches that of lepidocrocite $(\gamma-\mathrm{FeOOH})\left(2 \theta\right.$ angles of $27,36,47$, and $\left.60^{\circ}\right)$. A different pattern is observed at 1 and $1.8 \mathrm{~h}$ reaction time. These patterns do not correspond with any of the well-known iron oxide or oxyhydroxide phases. As the patterns are quite distinct, we speculate that they may represent the formation of an unknown intermediate phase. At $2 \mathrm{~h}$ of reaction time, most of the XRD peaks can be indexed to maghemite $\left(\gamma-\mathrm{Fe}_{2} \mathrm{O}_{3}\right)$, whose typical XRD profile is shown in red below the diffractograms. Additionally, there are two small and narrow peaks (at 24.6 and $47.2^{\circ}$ ) that reveal some remains of the intermediated phase (in correspondence with the observations by TEM). Figure S5 shows the XRD patterns of those samples collected after 6 months of storage. It is noteworthy that although sample NF0.5 is unstable and tends to evolve to ferroxyhyte $(\mathrm{FeOOH}$ JCPDS \#220353) over time when at room temperature the unknown intermediate phases (NF-1 and NF-1.8) remain stable after 6 months.

Figure $5 \mathrm{~b}$ shows the Mössbauer spectra of NF powders in the initial stages, $t=0.5-2 \mathrm{~h}$. The spectra of all four samples are magnetically split at $18 \mathrm{~K}$ and have been fitted with two or three sextets, with isomer shifts, $\delta$, around $0.50 \mathrm{~mm} / \mathrm{s}$, indicating that the samples consist of ferric phases. At $80 \mathrm{~K}$ and room temperature, doublets dominate the spectra. Again, the isomer shift of the doublets $(\sim 0.45 \mathrm{~mm} / \mathrm{s}$ at $80 \mathrm{~K}$ and $\sim 0.37 \mathrm{~mm} / \mathrm{s}$ at room temperature) as well as their quadrupole splittings $(\varepsilon \sim 0.6 \mathrm{~mm} / \mathrm{s})$ indicate only ferric phases in all four powder samples. The spectrum of NF- 0.5 obtained at $18 \mathrm{~K}$ has been fitted with three sextets.

The most dominating sextet (shown in dark blue color) has broad lines, a hyperfine field, $B_{\mathrm{hf}}$ around $45 \mathrm{~T}$, and $\varepsilon$ of around $0.0 \mathrm{~mm} / \mathrm{s}$. This sextet is most likely due to the presence of lepidocrocite, in agreement with the XRD and TEM results. The Neel temperature of lepidocrocite is $77 \mathrm{~K}^{32}$ but there appears to be quite a lot of magnetic relaxation of lepidocrocite already at low temperature, indicated by the broad lines and the blue sextet with the lower hyperfine field. At $80 \mathrm{~K}$ and room temperature, lepidocrocite is paramagnetic and hence seen as a doublet. The third sextet (orange) in the spectrum of sample NF-0.5 has sharp lines, a hyperfine field $B_{\text {hf }}$ of $\sim 50.6 \mathrm{~T}$, and a quadrupole shift, $\varepsilon$, of $-0.13 \mathrm{~mm} / \mathrm{s}$. The hyperfine parameters of this component match those of goethite, although this (probably nanocrystalline) phase was not detected by X-ray. At $80 \mathrm{~K}$, a ferric (goethite) sextet $\left(B_{\mathrm{hf}}=48.0 \mathrm{~T}, \varepsilon=-0.12 \mathrm{~mm} / \mathrm{s}\right)$ 
a)

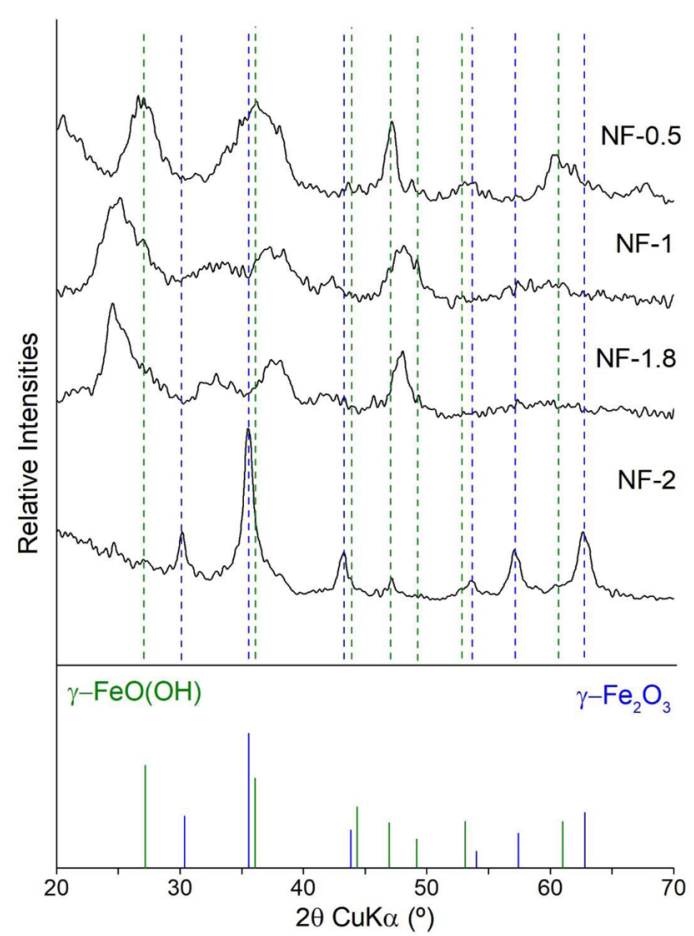

b)

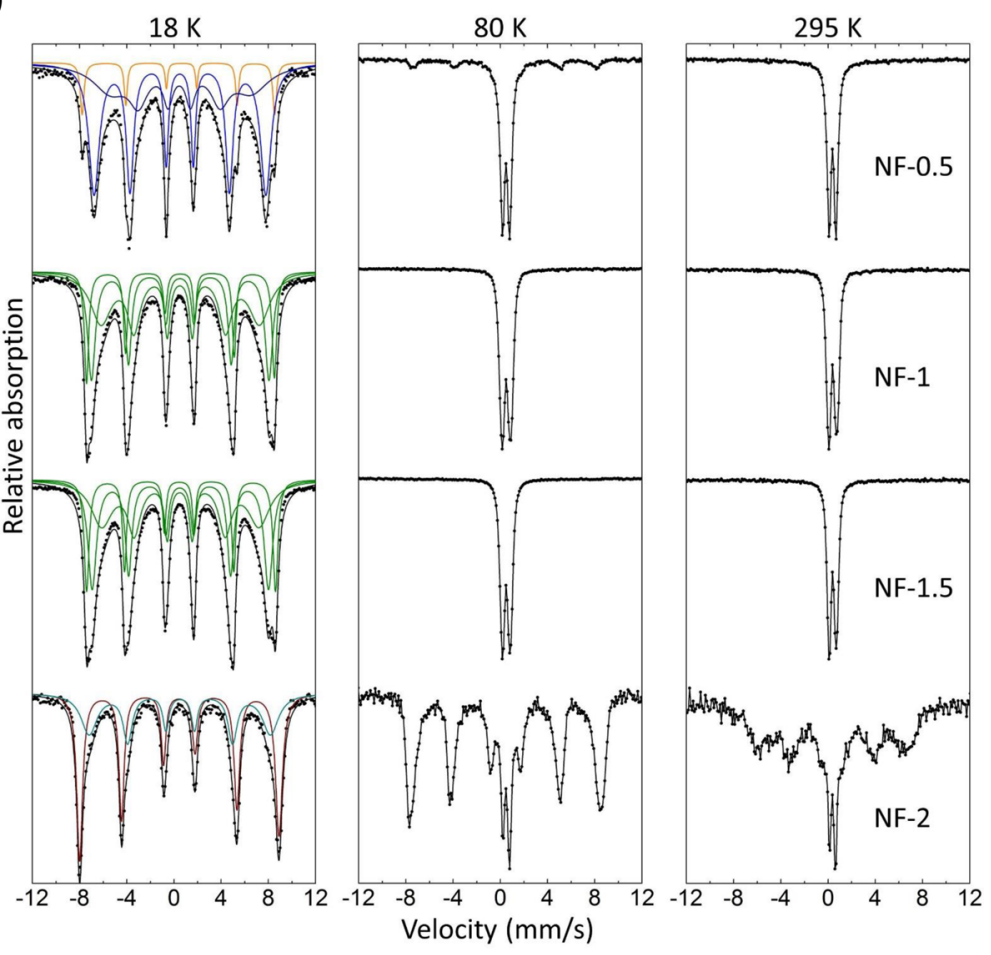

Figure 5. Formation mechanism of nanoflowers (NF) followed by: (a) X-ray diffraction. Expected peak positions of lepidocrocite $(\gamma$-FeOOH, green) and maghemite $\left(\gamma-\mathrm{Fe}_{2} \mathrm{O}_{3}\right.$, blue) are shown. (b) Mössbauer spectroscopy at 18, 80, and $295 \mathrm{~K}$. Samples were collected after $0.5,1,1.5,1.8$ and $2 \mathrm{~h}$ of aging time.
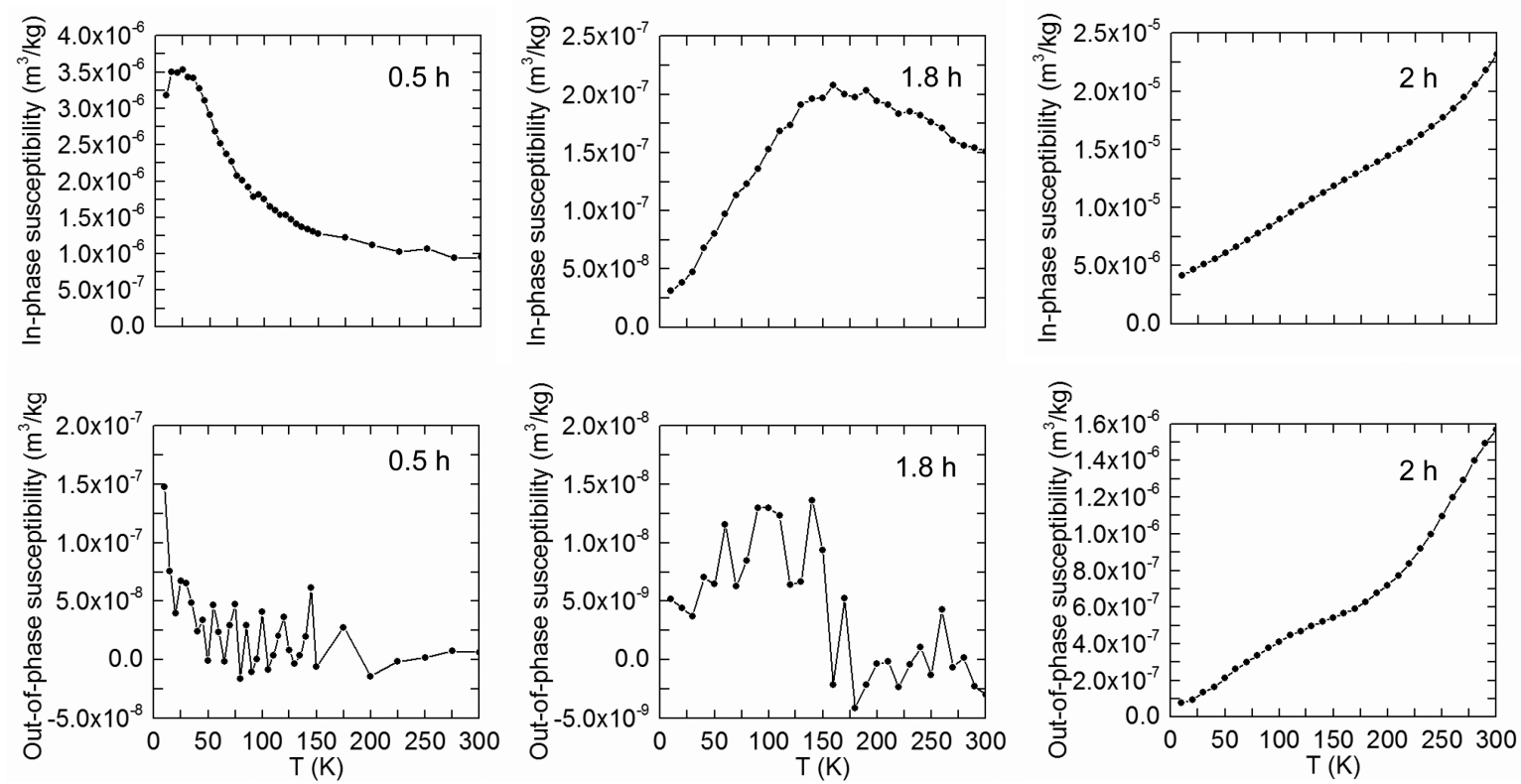

Figure 6. Temperature dependence of the low-field mass AC susceptibility at $11 \mathrm{~Hz}$ for the initial stages (heating times $0.5,1.8$, and $2 \mathrm{~h}$ ) of the nanoflower formation. Please note the different susceptibility scales in each plot.

with broad lines remains, whereas the room temperature spectrum features no goethite sextet, presumably due to superparamagnetic relaxation. This goethite component occupies about $7 \%$ of the spectral area of NF- 0.5 and is not seen in the other samples.

The Mössbauer spectra of NF- 1 and NF-1.5 are very similar to each other (in agreement with the XRD patterns of NF-1 and NF-1.8). These Mössbauer spectra, where the outer sextets have $B_{\mathrm{hf}} \sim 46.2-49.6 \mathrm{~T}, \delta \sim 0.5 \mathrm{~mm} / \mathrm{s}, \varepsilon \sim 0.0 \mathrm{~mm} / \mathrm{s}$, could be assigned to ferrihydrite or magnetically relaxing maghemite, but such assignment does not match the XRD patterns, whose main peaks are not found to match an existing phase (explicitly not ferrihydrite or maghemite). The Mössbauer parameters match well with those of an (new) undescribed intermediate phase in between that of lepidocrocite and maghemite, that is, a phase that is dehydroxylated lepidocrocite (or "maghemite with 
(a)

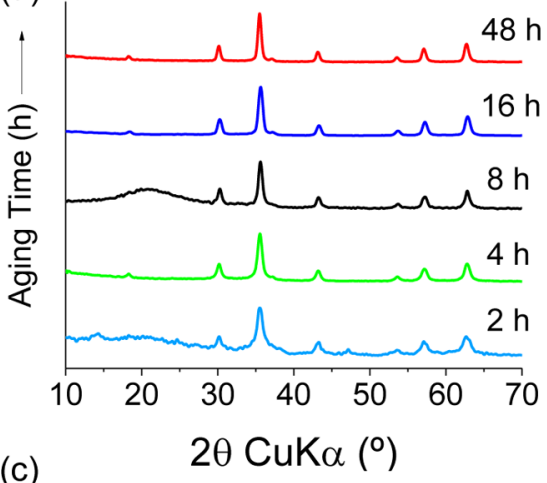

(b)

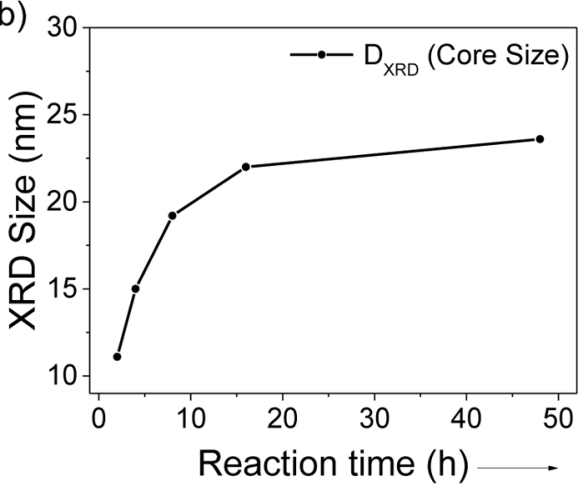

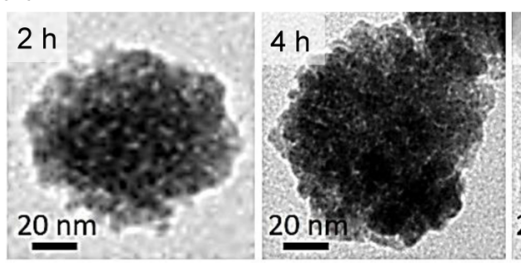
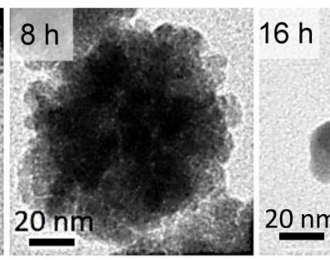

Figure 7. Structure of the NF along the formation at $t=2-48 \mathrm{~h}$ : (a) XRD patterns, (b) core size calculated by Scherrer's equation. (c) representative TEM images of the growing cores.

hydroxyl groups"). Mössbauer spectra obtained at $80 \mathrm{~K}$ in an applied field of $0.5 \mathrm{~T}$ show no magnetic splitting, indicating that this phase is paramagnetic at $80 \mathrm{~K}$. Recent studies have shown the presence of new intermediate phases that occur during the transformation between iron oxides/oxyhydroxides that may not be stable enough to be easily characterized. For example, an intermediate phase has been identified in the transformation between two-line ferrihydrite and hematite, which was termed as "hydromaghemite" or "ferriferrihydrite". 33 Although this phase has $B_{\mathrm{hf}}$ and $\delta$ values similar to those of the intermediate phase observed in this work, the X-ray patterns do not match, which means that we have captured a different "intermediate" phase.

The $18 \mathrm{~K}$ spectrum of NF-2 is dominated by a maghemite sextet (shown in wine red), with $B_{\mathrm{hf}}=52.6 .0 \mathrm{~T}, \varepsilon=0.01 \mathrm{~mm} / \mathrm{s}$, and $\delta \sim 0.5 \mathrm{~mm} / \mathrm{s}$. The asymmetry of this sextet (between lines one and six) and its isomer shift strongly indicate that it is ferric, that is, almost pure maghemite. ${ }^{34}$ At $80 \mathrm{~K}$, part of the spectrum $(\sim 20 \%)$ is relaxed into a doublet but the majority of the spectrum is a sextet with relatively sharp lines. Measurements obtained within an applied field (data not shown) show that the relaxed part is paramagnetic, that is, presumably due to the lepidocrocite or the intermediate phase. At room temperature, the sextet contribution remains most of its spectral area but has very broad lines. From this spectral behavior, a mixture of phases is assumed (in agreement with $\mathrm{XRD}$ ), that is, part of the sample is paramagnetic already at 80 $\mathrm{K}$ (lepidocrocite), whereas the remaining part of the sample (maghemite) shows slow relaxation even at room temperature.

AC magnetic measurements have been performed to follow the particle formation (Figure 6), especially at the initial stage of $0.5 \mathrm{~h}$, where the sample is unstable under the TEM beam. For NF-0.5, the in-phase susceptibility presents a Curie-like behavior down to $25 \mathrm{~K}$, with a magnetic effective moment per iron ion of about $2.6 \mu_{\mathrm{B}}$ (Bohr magnetons), calculated by assuming, for example, the formula of lepidocrocite. The deviation of the in-phase susceptibility from the Curie law behavior at low temperatures together with the rise of the outof-phase component suggests that magnetic blocking of very small nanoparticles takes place below $\sim 20 \mathrm{~K}$. For NF-1.8, the maximum of the in-phase component together with the out-ofphase component step at $\sim 150 \mathrm{~K}$ indicates magnetic blocking of nanoparticles up to this temperature. The location of these maxima, which are at higher temperatures than in the case of NF-0.5, indicates the growth of the nanoparticles at these early stages. Given that at this temperature, lepidocrocite and the intermediate phase of NF-1 and NF-1.5 should be paramagnetic, this feature may be caused by particles with considerable magnetization, possibly being made of the undescribed intermediate phase in its advance stage $(1.8 \mathrm{~h})$. For NF-2, the appearance of both components of the AC susceptibility is typical of particles magnetically blocked well above the room temperature, displaying susceptibility maxima at temperatures above the measured range, in agreement with the observation of maghemite flowers $(\sim 60 \mathrm{~nm})$ by TEM.

The results of TEM, XRD, Mössbauer spectroscopy, and AC susceptibility support that prior to formation of maghemite nanoflowers, lepidocrocite with a laminar habit is formed, which transforms to maghemite through an intermediate phase, probably that of dehydroxylated lepidocrocite.

Consequently, the formation mechanism is proposed to occur as follows at this initial stage

$$
\begin{aligned}
& \mathrm{CH}_{3} \mathrm{COO}^{-}+\mathrm{H}_{2} \mathrm{O} \rightarrow \mathrm{CH}_{3} \mathrm{COOH}+\mathrm{OH}^{-} \\
& \mathrm{Fe}^{3+}+3 \mathrm{OH}^{-} \rightarrow \mathrm{Fe}(\mathrm{OH})_{3} \\
& 2 \mathrm{Fe}(\mathrm{OH})_{3} \rightarrow 2 \gamma-\mathrm{FeOOH}+\mathrm{H}_{2} \mathrm{O} \\
& \mathrm{CH}_{3} \mathrm{OH}-\mathrm{CH}_{2} \mathrm{OH} \rightarrow \mathrm{CH}_{3}-\mathrm{CHO}+\mathrm{H}_{2} \mathrm{O}
\end{aligned}
$$

First, NaAc causes a weak hydrolyzation (eq 1), which controls the release rate of $\mathrm{OH}^{-}$. It has been reported that iron ions could coordinate with the acetate anion $\left(\mathrm{CH}_{3} \mathrm{COO}^{-}\right)$, forming a coordination compound that could evolve to magnetite directly in the presence of EG that is oxidized to glycolaldehyde (eq 4), a reductant capable of reducing most noble metal ions. ${ }^{35}$ However, in our case, because lepidocrocite and dehydroxylated lepidocrocite have been suggested through 
(a)
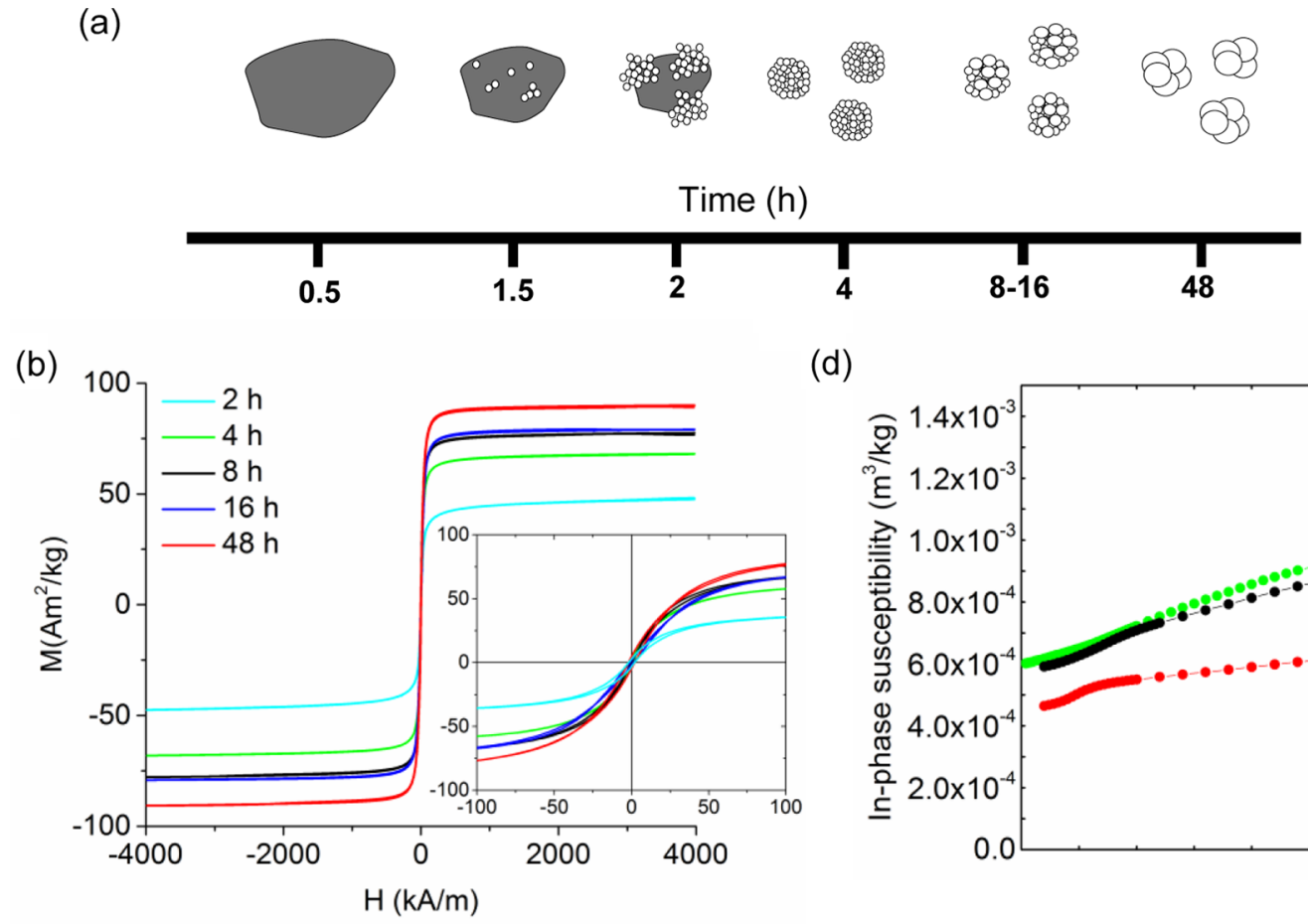

(d)

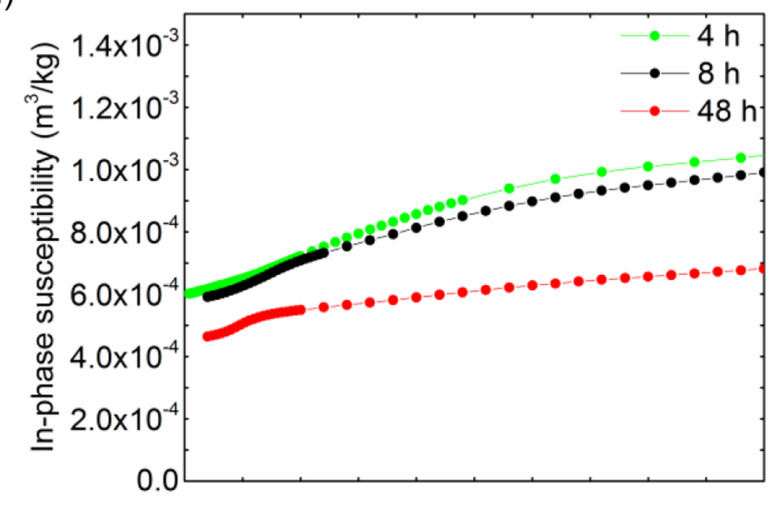

(c)

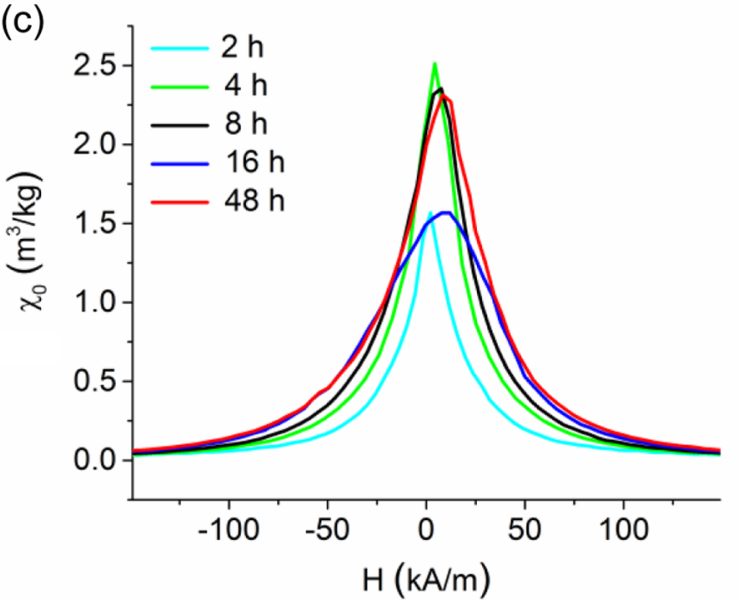

(e)

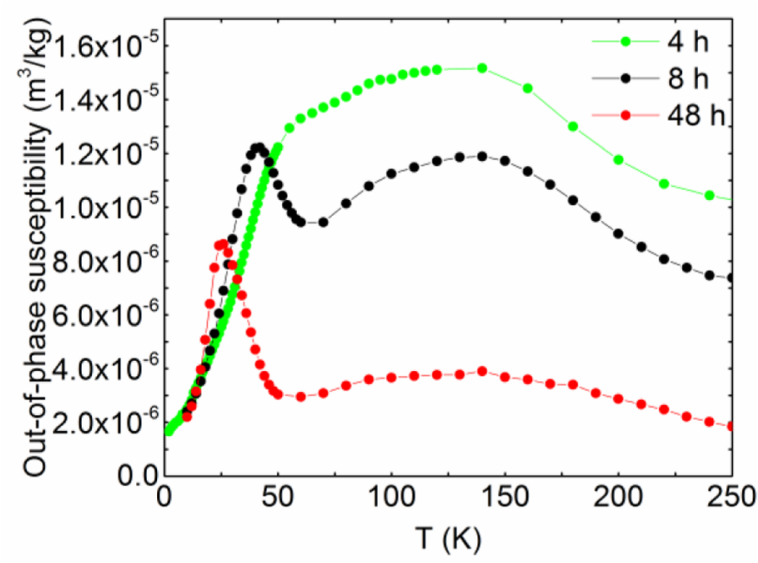

Figure 8. Formation mechanism of maghemite nanoflowers: (a) sketch of particle evolution throughout the aging process, (b) DC magnetization curves at $290 \mathrm{~K}$ of samples NF-2-48, (c) mass initial susceptibility $\left(\chi_{0}\right)$ of samples NF-2-48, and (d, e) temperature dependence of the low-field AC susceptibility at $11 \mathrm{~Hz}$, along the formation of nanoflowers at 4,8 , and $48 \mathrm{~h}$ of aging time.

$\mathrm{XRD}$ and Mössbauer spectroscopy as intermediate phases (samples NF-0.5-1.8), we propose that the mechanism follows a sol-gel reaction, where the initial $\gamma$-FeOOH nuclei are formed thanks to the sodium acetate releasing $\mathrm{OH}^{-}$(eqs 2 and 3 ). The high temperatures, pressure, and the action of sodium acetate allow the formation of lepidocrocite by a burst type nucleation, ${ }^{36,37}$ which in this case seems to occur rapidly $(<0.5$ h). After nucleation, there is phase transformation (via dehydrolyzation) and aggregation, leading to maghemite nanoflowers with a fixed size. To figure out whether the immediate product is magnetite or maghemite is by no means a trivial issue. On one hand, a reduction process (eq 4) can occur during the solvent-mediated transformation to magnetite ${ }^{2}$ $\left(2 \mathrm{FeO}(\mathrm{OH})+\mathrm{Fe}(\mathrm{OH})_{2} \rightarrow \mathrm{Fe}_{3} \mathrm{O}_{4}+2 \mathrm{H}_{2} \mathrm{O}\right)$, which is easily oxidized maghemite. On the other hand, it has been demonstrated by Navrotsky et al. ${ }^{38}$ that a direct size-driven transformation of lepidocrocite to maghemite is thermodynamically possible $\left(2 \mathrm{FeO}(\mathrm{OH}) \rightarrow \gamma-\mathrm{Fe}_{2} \mathrm{O}_{3}+\mathrm{H}_{2} \mathrm{O}\right)$. Our results seem to support the thermodynamic considerations stated by Navrotsky, and we conclude that this oxyhydroxide transforms directly to maghemite, ${ }^{39}$ but we could not exclude the initial presence of small amounts of $\mathrm{Fe}^{2+}$ in our samples that will remain undetectable by further oxidation during the characterization process.

Aging Process: Crystal Growth $(2-48$ h). Once the maghemite nanoflowers have formed, after $2 \mathrm{~h}$ of reaction time, an aging process starts. This process has been studied through TEM, XRD, and magnetic measurements. Figure 7a shows the XRD patterns collected for NF-2-48. After $4 \mathrm{~h}$, only maghemite is detected. The diffraction peaks become narrower throughout the aging process, and the crystal size obtained from the width of the diffraction peaks by use of the Scherrer formula shows that the nanoflowers undertake a crystallization process, increasing its mean crystal size $d_{\mathrm{XRD}}$ from 11 to $23 \mathrm{~nm}$ (Figure $7 \mathrm{~b}$ ). A similar trend is observed by TEM, where the cores of the particles are seen to grow over time (Figure 7c). 
Throughout the time, the organic content of the samples is reduced (thermogravimetric analysis (TGA) is provided in Figure S7) from 12 to $4 \%$.

Figure $8 \mathrm{a}$ illustrates the whole progress of the formation mechanism and crystallization of NF samples, which can be linked to eqs 1-4, where laminar lepidocrocite is formed and then transformed to maghemite nanoflowers. This has been characterized magnetically through direct current (DC) magnetometry and AC susceptibility. The magnetic properties of the nanoflowers during the aging steps from 2 to $48 \mathrm{~h}$ were evaluated in detail (Figure $8 \mathrm{~b}-\mathrm{e}$ ). By prolonging the aging time $(4,8,16$, and $48 \mathrm{~h})$, the nanoflowers' saturation magnetization is increased from 48 to $90 \mathrm{~A} \mathrm{~m}^{2} / \mathrm{kg}$ at $290 \mathrm{~K}$ (Figure $8 \mathrm{~b}$ ), which implies quite a significant enhancement. The samples also show increasing coercivity values $(\sim 0.5-2 \mathrm{kA} / \mathrm{m}$ at $\mathrm{RT}$ and $8-19$ $\mathrm{kA} / \mathrm{m}$ at $5 \mathrm{~K}$ ) with aging. The initial mass susceptibility at low fields $\left(\chi_{0}\right)$ was calculated for all samples through DC magnetometry from the numerical field derivative of the magnetization (Figure $8 \mathrm{c})\left(\chi_{0}\right.$ as calculated in Table 2$)$. Samples NF-4, NF-8, and NF-48 have $\chi_{0}$ values of $2.3-2.5 \mathrm{~m}^{3} /$ $\mathrm{kg}$, which is higher than those of SC and HS nanoparticles $\left(\chi_{0}\right.$ for these samples is 1.7 and $1.35 \mathrm{~m}^{3} / \mathrm{kg}$, respectively). This enhancement of the magnetic susceptibility has been previously linked to a magnetic cooperative effect due to aggregation of cores forming densely packed nanoflowers. ${ }^{12}$ The magnetic behavior of the progressively aged nanoflowers has also been investigated by means of AC susceptibility (see Figure $8 \mathrm{~d}, \mathrm{e}$ ). The results can be interpreted by considering three contributions: (i) a positive in-phase susceptibility level at room temperature due to the presence of rather large magnetic entities with permanent magnetism, (ii) a wide and rounded out-of-phase maximum, very likely indicating the blocking of magnetic entities of intermediate size, and (iii) a lowtemperature step in the in-phase component accompanied by a rather sharp peak in the out-of-phase below $\sim 50 \mathrm{~K}$. The major contribution corresponds to the particles magnetically blocked well above room temperature, already detected in sample NF-2, although from $t=4$ to $48 \mathrm{~h}$, the magnitude of the resulting in-phase susceptibility is significantly higher because paramagnetic lepidocrocite dissolves in favor of other strong magnetic phases. These samples present a much higher signal per mass of the sample than NF-2 (Figure 6), in agreement with the disappearance of the lepidocrocite or the intermediate phases observed by TEM and XRD at the longest reaction times. For NF-4, NF-8, and NF-48, the magnitude of the room temperature in-phase component decreases for increasing aging time. This result well agrees with the field derivative of magnetization shown in Figure 8c. We believe this value mostly results from the contribution of the large magnetic entities. The magnetic dynamics of the lower temperature anomalies have been investigated to interpret their nature (detailed discussion, Figure S8 and Table S1 from the Supporting Information (SI)). The relative sharpness of the lowest temperature peaks, their pre-exponential factors of the Arrhenius law, their regular dependence with the aging time, and the occurrence also in iron oxide nanoparticles prepared by completely different methods $^{40,41}$ point to some phenomenon intrinsic to maghemite or to some usually appearing intermediate phase, although no rigorous interpretation of this phenomenon can be offered yet.

In summary, the analysis of the samples with longest reaction times indicates that lepidocrocite and the "undescribed" intermediate phase have disappeared. Interestingly, increasing reaction times give rise to a significant increase of the core sizes but not of the flower size.

Biomedical Applications. We here evaluate aspects relevant for biomedical applicability (especially colloidal stability and heating potential for magnetic hyperthermia) of four selected samples: SC, HS, NF-4, and NF-48, as these samples represent distinct different morphologies (tuned by the $\mathrm{NaAc}$ concentration) and hold different core sizes. The surface charge and the colloidal stability of samples SC, HS, NF-4, and NF-48 were enhanced by citric acid coating. Infrared spectroscopy confirms the successful citric acid coating from the absorption bands typical of the carboxyl at 1384 and 1022 $\mathrm{cm}^{-1}$ (Figure $\mathrm{S} 6$ of $\mathrm{SI}$ ). The hydrodynamic size $\left(D_{\mathrm{h}}\right)$, polydispersity index (PdI), and isoelectric points (IEPs) of as-synthesized and coated particles are summarized in Table 4.

Table 4. Colloidal Properties of Maghemite Nanoparticles with the Different Morphologies ${ }^{a}$

\begin{tabular}{lccccccc} 
& \multicolumn{3}{c}{ as-synthesized } & & \multicolumn{3}{c}{ citric acid coating } \\
\cline { 2 - 3 } \cline { 7 - 8 } \multicolumn{1}{c}{ sample } & $D_{\mathrm{h}}(\mathrm{nm})$ & PdI & IEP & & $D_{\mathrm{h}}(\mathrm{nm})$ & PdI & IEP \\
SC-CIT & 896 & 0.28 & 5.5 & & 161 & 0.26 & 5.0 \\
HS-CIT & 705 & 0.31 & 5.7 & & 298 & 0.25 & 5.1 \\
NF-4-CIT & 331 & 0.27 & 6.6 & & 109 & 0.14 & 4.9 \\
NF-48-CIT & 965 & 0.23 & 6.0 & & 102 & 0.11 & 4.0
\end{tabular}

${ }^{a}$ Single-core (SC), hollow spheres (HSs), and nanoflowers at different aging times (NF-4 and NF-28) coated with citric acid (CIT).

The hydrodynamic sizes are smaller for the coated samples than for the as-synthesized particles due to an increase in the surface charge, as the shift of the IEP to lower pHs confirms (the NPs have increased surface charge at $\mathrm{pH} 7$ ). Figure 9a shows the change in hydrodynamic size after the coating for sample NF-4. $D_{\mathrm{h}}$ for NF-4 changes from a polydispersed distribution to a narrow monomodal distribution centered at $\sim 100 \mathrm{~nm}$ after the coating, which is closer to the average particle size determined by TEM (Table 1). Furthermore, number-weighted $D_{\mathrm{h}}$ values for NF samples $(50-70 \mathrm{~nm})$ do approach $d_{\text {TEM }}$, as this is number weighted. Similarly, a change to a more well-dispersed system is also seen for sample NF-48 when coated with citric acid. For samples SC and HS, the hydrodynamic size after coating is 161 and $298 \mathrm{~nm}$, respectively, which is much larger than $d_{\text {TEM }}$ for sample SC and relatively larger than $d_{\text {TEM }}$ for sample HS, suggesting a certain degree of agglomeration of the particles in suspension.

Figure $9 \mathrm{~b}$ shows the measured $\zeta$ potential curve for sample NF-4. The citric acid coating promotes the stabilization by repulsive forces ${ }^{42}$ because there is an increase of the surface charge from -10 up to $-40 \mathrm{mV}$ at $\mathrm{pH} 7$, assuring long-term stability at physiological $\mathrm{pH}$. We observe a similar increase of surface charge for samples SC, HS, and NF-48. Moreover, there is, for all samples, a shift of the isoelectric point (zero $\zeta$ potential) to lower $\mathrm{pH}$ values that confirms the successful coating with carboxyl groups for sample NF-4. This shift is larger in the nanoflowers, probably due to the higher surface-tovolume ratio in comparison with the single-core (SC) particles and the hollow spheres (HSs).

The heating capacity of the coated materials in water at physiological $\mathrm{pH}$ was evaluated to study the differences in terms of sample morphology and crystallinity. Figure 10 shows the different specific loss power (SLP) values calculated through "the slope method" (change of temperature over time for different frequencies: 419,542 , and $710 \mathrm{kHz}$ ), for 

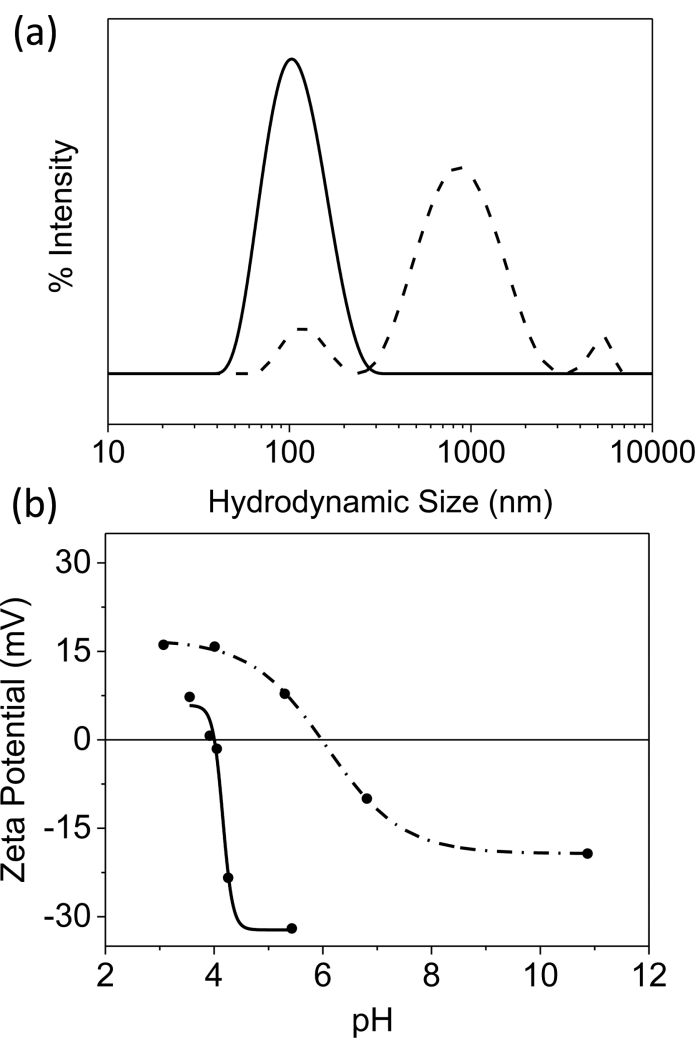

Figure 9. Effect of the citric acid (CA) coating on sample NF-4: (a) DLS measurements and (b) $\zeta$ potential curves fitted to a Boltzmann sigmoidal function. Dashed line connects data points for the assynthesized sample. The continuous line connects data points for citric acid coating.

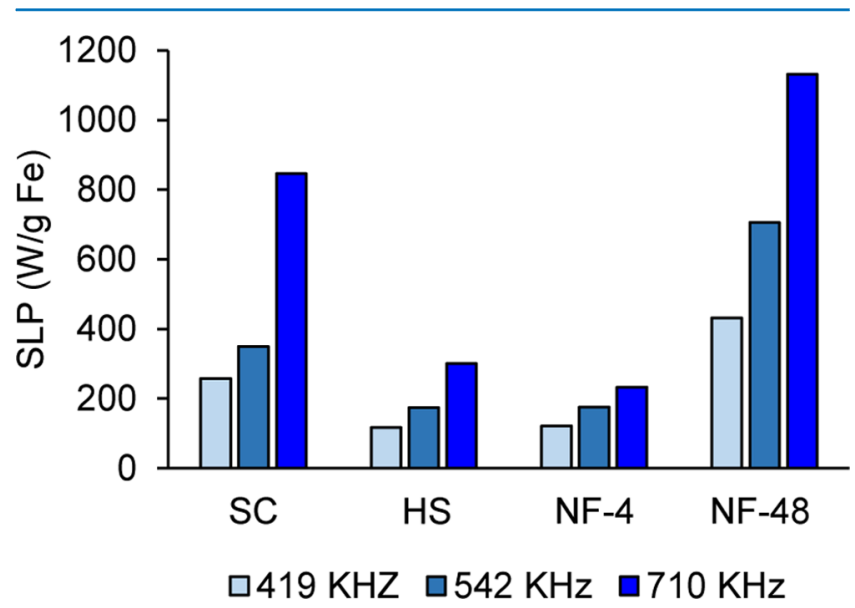

Figure 10. SLP values of samples of citric acid (CA)-coated SC, HS, NF-4, and NF-48. SLP values of different samples calculated by the slope method. The field amplitude was $23.8 \mathrm{kA} / \mathrm{m}$ and three different frequencies were applied: 419,542 , and $710 \mathrm{kHz}$.

samples SC, HS, NF-4, and NF-48. The specific loss power is found as

$$
\mathrm{SLP}(\mathrm{W} / \mathrm{g} \mathrm{Fe})=c \times \frac{\text { mass }_{\text {sample }}}{\text { mass }_{\mathrm{Fe}}} \times \frac{\Delta T}{\Delta t}
$$

where $c$ is the capacity of the suspension (typically assumed to be the heat capacity of the suspension medium for low concentrations of MNPs). ${ }^{43}$ Nanoflowers with a core size of 23 $\mathrm{nm}$ (NF-48) have the highest SLP values, for example, yielding
1131.2 W/g Fe for a frequency of $710 \mathrm{kHz}$. This sample displays SLP values nearly 5 times larger than those of nanoflowers with crystal size of $15 \mathrm{~nm}$ (NF-4), nearly 4 times larger than hollow spheres and 1.5 times larger than single-core nanoparticles, although these two latter samples (HS and SC) have bigger core sizes of 27 and $36 \mathrm{~nm}$, respectively. This demonstrates that both core sizes and the packing of the cores have decisive influence on the heating capacity. Specifically, we observe that nanoflowers with large cores (controlled by annealing) and densely packed cores (tuned by $\mathrm{NaAc}$ concentration) offer enhanced heating capacity compared with nanoflowers with smaller crystallite sizes, hollow spheres, and single-core nanoparticles used in this work.

Additional SAR measurements have been performed in viscous media (agar 2\%). Although the obtained heating capacity for the SC and HS samples does not change depending on the nanoparticle surrounding media, samples NF- 4 and NF48 present a significant change depending on the solvent. The behavior of samples NF-4 and NF-48 resembles the one observed before for similar magnetite nanoflowers, with a lower performance in viscous media. ${ }^{15}$ It is remarkable that whereas the heating performance of sample NF-48 drastically decreased $(37 \%)$, in the case of sample NF-4, the fall was slight (11\%). This result suggests how the difference in the core size (23 vs $15 \mathrm{~nm}$ for maghemite NPs and thus in the range of the transition from the ferromagnetic to the superparamagnetic regime) affects the SAR when comparing fairly similar overall clusters.

Given the heterogeneity in the conditions (field and frequency) used to measure SLP by different research groups, and the multiple possibilities of the materials characterized (with different particle size, shape, structure (single-core or multicore), crystalline structure, composition, etc.), it is complicated to find the most appropriate examples in the literature to compare our data. For example, $30 \mathrm{~nm}$ single-core magnetite nanoparticles, obtained by $\mathrm{FeSO}_{4}$ precipitation and subsequent ageing, had an SLP value of $95 \mathrm{~W} / \mathrm{g}$ (field amplitude $10 \mathrm{kA} / \mathrm{m}$ and frequency of $249 \mathrm{kHz}){ }^{44}$ Our SC sample, with a similar particle size, presents a higher SLP value $(257 \mathrm{~W} / \mathrm{g})$ with the lowest amplitude and frequency $(22.8 \mathrm{kA} /$ $\mathrm{m}$ and $419 \mathrm{kHz}$, respectively); however, the measurement conditions are not exactly the same. Regarding hollow structures, liposomes $(120 \mathrm{~nm})$ encapsulating magnetite particles (10 nm core size) presented an SLP value of 210 $\mathrm{W} / \mathrm{g}{ }^{15}$ With the same conditions, the sample HS has a similar SLP value of $301 \mathrm{~W} / \mathrm{g}$, although the core size and the particle size are not exactly the same. In the frame of multicore structures, materials obtained by coprecipitation of ferric and ferrous chloride in a microwave, with a crystal size of $13 \mathrm{~nm}$ and hydrodynamic sizes of $123 \mathrm{~nm}$, similar to sample NF-4, had an SLP value of $190 \mathrm{~W} / \mathrm{g}$ (field amplitude $10.5 \mathrm{kA} / \mathrm{m}$ and frequency of $950 \mathrm{kHz}){ }^{45}$ These values are similar to the ones obtained for sample NF-4 at the most similar conditions measured in our case $(232 \mathrm{~W} / \mathrm{g}$ with field amplitude $24 \mathrm{kA} / \mathrm{m}$ and frequency of $700 \mathrm{kHz}$ ). Measurements in the same field and frequency conditions for a material similar to sample NF48 (magnetite nanoflowers of $22 \mathrm{~nm}$ obtained by the polyolmediated synthesis) yielded similar SLP data $(1130 \mathrm{~W} / \mathrm{g}$ for $\mathrm{NF}-48$ and $1180 \mathrm{~W} / \mathrm{g}$ for the $22 \mathrm{~nm}$ nanoflowers). The obtained SLP data for our materials envisages a possible use for magnetic fluid hyperthermia treatment of cancer; however, the lack of standardized measurement conditions makes it complicated to compare the data with other existing materials. 
The intrinsic loss power (ILP) of the samples was calculated from eq 6

$$
\operatorname{ILP}\left(\mathrm{nHm}^{2} / \mathrm{kg}\right)=\frac{\mathrm{SLP}}{H^{2} f}
$$

It ranges from 0.5 to 0.6 (in the case of samples NF-4 and HS, respectively), 1.9 (sample SC), up to 2.6 (sample NF-48). Lastly, it must be noted that the product $H \cdot f$ was kept for all combinations below $4.85 \times 10^{8} \mathrm{kA} / \mathrm{m} \mathrm{s}$, which is mandatory for avoiding nonspecific eddy heating in tissues. ${ }^{46}$

\section{CONCLUSIONS}

The polyol-mediated synthesis has been explored and developed for the preparation of well-controlled magnetic nanoparticles with different core sizes and arrangement to form the final single-core and multicore particles. The particles are formed by burst nucleation and growth processes that determine the final nanostructure going from single-core to hollow spheres and nanoflowers, with high crystallinity, due to the selected polyol synthesis route that uses high temperature over long periods of time.

Sodium acetate is found to be a key parameter governing the self-assembly process. In this reaction, it has a double role: the particle formation and the nucleation and growth. For higher acetate concentration, massive nucleation and growth by diffusion takes place, resulting in single-core particles. As the amount of acetate is reduced, the initial nanocrystalline subunits seem to grow by partial aggregation in hollow spheres. Further reduction in acetate leads to initial nanocrystalline nuclei that strongly aggregate to produce the final flower-like particles.

The synthesis of magnetic nanoflowers occurs via burst nucleation, growth by aggregation, and recrystallization that takes place over time. The initial stages of the reaction are composed of lepidocrocite, which suffers a fast dehydroxylation, transforming to an intermediate undescribed phase, possibly a partly dehydroxylated lepidocrocite, which evolves to maghemite nanoflowers. A prolonged heating of the flowers leads to nanoflower particles with larger cores with interesting magnetic and colloidal properties and consequently high heating capacities, sample NF-48 being the one that displays the highest ILP value.

In the future, the transferred energy from an exciting magnetic field to nanoparticles, leading to a dissipation of heat in targeted bodies such as tumors, is likely to be explored much further. Taking advantage of this physical phenomenon, magnetic nanoparticles are also considered by many as potential chemotherapy and radiotherapy enhancement agents, where a limited heat dissipation dose increases the effectiveness in cell destruction. Our studies have outlined possible ways to control the formation of distinct structures via polyol synthesis, specifically the core sizes and arrangement have been tuned. This indicates ways to tailor and optimize magnetic properties for specific applications. On one hand, the most wellcrystallined nanoflowers (NF-48) have the highest heating capacity. On the other hand, nanoflowers with core size above $15 \mathrm{~nm}$ (NF-4) combine high saturation magnetization and initial susceptibility, while conserving low remanence at room temperature, which can be suitable for specific biomedical applications and also applications such as magnetic separation.

\section{ASSOCIATED CONTENT}

\section{Supporting Information}

The Supporting Information is available free of charge on the ACS Publications website at DOI: 10.1021/acsomega.7b00975.

Transmission electron microscopy images of hollow particles; the nanoparticles obtained in the absence of PVP and log-normal size distributions of samples (NF4-48); infrared spectra of magnetite nanoparticles before and after the coating process; thermogravimetric analysis for different periods of crystallization: 2, 4, 8, and $48 \mathrm{~h}$; AC magnetic susceptibility analysis of the magnetic dynamics of samples above $2 \mathrm{~h}$ (PDF)

\section{AUTHOR INFORMATION}

\section{Corresponding Authors}

*E-mail: helena_gr@icmm.csic.es (H.G.).

*E-mail: puerto@icmm.csic.es (M.P.M.)

*E-mail: lu@unizar.es (L.G.).

ORCID

Sabino Veintemillas-Verdaguer: 0000-0002-3015-1470

M. Puerto Morales: 0000-0002-7290-7029

Lucía Gutiérrez: 0000-0003-2366-3598

\section{Author Contributions}

The manuscript was written through contributions of all authors. All authors have given approval to the final version of the manuscript.

\section{Funding}

This work was supported by the EC FP-7 grant "NanoMag" (grant agreement no. 604448) and the Spanish Government by MAGO project (MAT2014-52069-R).

\section{Notes}

The authors declare no competing financial interest.

\section{ACKNOWLEDGMENTS}

L.G. acknowledges financial support from the Ramón y Cajal subprogram (RYC-2014-15512) and M.E.F.B. acknowledges financial support from the Brazilian agency $\mathrm{CNPq}$ grant (232947/2014-7). Servicio General de Apoyo a la Investigación-SAI, Universidad de Zaragoza is acknowledged. Support Laboratories at ICMM, microscopy service at CNB, and Servicio Interdepartamental de Investigación (Sidi), Universidad Autonoma de Madrid are also acknowledged.

\section{ABBREVIATIONS}

NaAc, sodium acetate; PVP, poly(vinylpyrrolidone); EG, ethylene glycol; SC, single-core; HSs, hollow spheres; NFs, nanoflowers; TEM, transmission electron microscopy; XRD, Xray diffraction; DLS, dynamic light scattering; FTIR, Fourier transform infrared spectroscopy; TGA, thermogravimetric analysis; SAR, specific absorption rate; SLP, specific loss power; ILP, intrinsic loss power; $M_{\mathrm{s}}$, saturation magnetization; $\chi_{0}$, initial mass susceptibility at low fields; $H_{\mathcal{O}}$ coercivity; $M_{\mathrm{r}}$, remanence

\section{REFERENCES}

(1) Fievet, F.; Lagier, J.; Blin, B.; Beaudoin, B.; Figlarz, M. Homogeneous and heterogeneous nucleations in the polyol process for the preparation of micron and submicron size metal particles. Solid State Ionics 1989, 32-33, 198-205. 
(2) Dong, H.; Chen, Y.-C.; Feldmann, C. Polyol synthesis of nanoparticles: status and options regarding metals, oxides, chalcogenides, and non-metal elements. Green Chem. 2015, 17, 4107-4132.

(3) Qu, H.; Caruntu, D.; Liu, H.; O'Connor, C. J. Water-dispersible iron oxide magnetic nanoparticles with versatile surface functionalities. Langmuir 2011, 27, 2271-2278.

(4) Cai, W.; Wan, J. Facile synthesis of superparamagnetic magnetite nanoparticles in liquid polyols. J. Colloid Interface Sci. 2007, 305, 366370.

(5) Haynes, W. Handbook of Chemistry and Physics, 93rd ed.; Chemical Rubber Company, 2012.

(6) Zhang, H.; Watanabe, T.; Okumura, M.; Haruta, M.; Toshima, N. Catalytically highly active top gold atom on palladium nanocluster. Nat. Mater. 2011, 11, 49-52.

(7) Soumare, Y.; Garcia, C.; Maurer, T.; Chaboussant, G.; Ott, F.; Fiévet, F.; Piquemal, J.-Y.; Viau, G. Kinetically Controlled Synthesis of Hexagonally Close-Packed Cobalt Nanorods with High Magnetic Coercivity. Adv. Funct. Mater. 2009, 19, 1971-1977.

(8) Gutiérrez, L.; Costo, R.; Grüttner, C.; Westphal, F.; Gehrke, N.; Heinke, D.; Fornara, A.; Pankhurst, Q. A.; Johansson, C.; VeintemillasVerdaguer, S.; Morales, M. P. Synthesis methods to prepare single- and multi-core iron oxide nanoparticles for biomedical applications. Dalton Trans. 2015, 44, 2943-2952.

(9) Cheng, C.; Xu, F.; Gu, H. Facile synthesis and morphology evolution of magnetic iron oxide nanoparticles in different polyol processes. New J. Chem. 2011, 35, 1072-1079.

(10) Caruntu, D.; Caruntu, G.; Chen, Y.; O'Connor, C. J.; Goloverda, G.; Kolesnichenko, V. L. Synthesis of variable-sized nanocrystals of $\mathrm{Fe}_{3} \mathrm{O}_{4}$ with high surface reactivity. Chem. Mater. 2004, 16, 5527-5534.

(11) Laurent, S.; Forge, D.; Port, M.; Roch, A.; Robic, C.; Elst, L. V.; Muller, R. N. Magnetic iron oxide nanoparticles: synthesis, stabilization, vectorization, physicochemical characterizations, and biological applications. Chem. Rev. 2008, 108, 2064-2110.

(12) Lartigue, L.; Hugounenq, P.; Alloyeau, D.; Clarke, S. P.; Lévy, M.; Bacri, J.-C.; Bazzi, R.; Brougham, D. F.; Wilhelm, C.; Gazeau, F. Cooperative organization in iron oxide multi-core nanoparticles potentiates their efficiency as heating mediators and MRI contrast agents. ACS Nano 2012, 6, 10935-10949.

(13) Kim, B. H.; Lee, N.; Kim, H.; An, K.; Park, Y. I.; Choi, Y.; Shin, K.; Lee, Y.; Kwon, S. G.; Na, H. B.; et al. Large-scale synthesis of uniform and extremely small-sized iron oxide nanoparticles for highresolution T1 magnetic resonance imaging contrast agents. J. Am. Chem. Soc. 2011, 133, 12624-12631.

(14) Sun, Q.; Ren, Z.; Wang, R.; Chen, W.; Chen, C. Magnetite hollow spheres: solution synthesis, phase formation and magnetic property. J. Nanopart. Res. 2011, 13, 213-220.

(15) Di Corato, R.; Espinosa, A.; Lartigue, L.; Tharaud, M.; Chat, S.; Pellegrino, T.; Ménager, C.; Gazeau, F.; Wilhelm, C. Magnetic hyperthermia efficiency in the cellular environment for different nanoparticle designs. Biomaterials 2014, 35, 6400-6411.

(16) Mahmoudi, M.; Hofmann, H.; Rothen-Rutishauser, B.; PetriFink, A. Assessing the in vitro and in vivo toxicity of superparamagnetic iron oxide nanoparticles. Chem. Rev. 2012, 112, 23232338.

(17) Weissleder, R.; Stark, D. D.; Engelstad, B. L.; Bacon, B. R.; Compton, C. C.; White, D. L.; Jacobs, P.; Lewis, J. Superparamagnetic iron oxide: pharmacokinetics and toxicity. AJR, Am. J. Roentgenol. 1989, 152, 167-173.

(18) Pankhurst, Q. A.; Connolly, J.; Jones, S. K.; Dobson, J. Applications of magnetic nanoparticles in biomedicine. J. Phys. D: Appl. Phys. 2003, 36, R167-R181.

(19) Gavilán, H.; Kowalski, A.; Heinke, D.; Sugunan, A.; Sommertune, J.; Varón, M.; Bogart, L. K.; Posth, O.; Zeng, L.; González-Alonso, D.; Balceris, C.; Fock, J.; Wetterskog, E.; Frandsen, C.; Gehrke, N.; Grüttner, C.; Fornara, A.; Ludwig, F.; VeintemillasVerdaguer, S.; Johansson, C.; Morales, M. P. Colloidal Flower-shaped Iron Oxide Nanoparticles: Synthesis Strategies and Coatings. Part. Part. Syst. Charact. 2017, No. 1700094.
(20) Gavilán, H.; Posth, O.; Bogart, L. K.; Steinhoff, U.; Gutiérrez, L.; Morales, M. P. How shape and internal structure affect the magnetic properties of anisometric magnetite nanoparticles. Acta Mater. 2017, $125,416-424$.

(21) Martina, M.-S.; Fortin, J.-P.; Ménager, C.; Clément, O.; Barratt, G.; Grabielle-Madelmont, C.; Gazeau, F.; Cabuil, V.; Lesieur, S. Generation of superparamagnetic liposomes revealed as highly efficient MRI contrast agents for in vivo imaging. J. Am. Chem. Soc. 2005, 127, 10676-10685.

(22) Luo, W.; Ma, H.; Mou, F.; Zhu, M.; Yan, J.; Guan, J. StericRepulsion-Based Magnetically Responsive Photonic Crystals. Adv. Mater. 2014, 26, 1058-1064.

(23) Hachani, R.; Lowdell, M.; Birchall, M.; Hervault, A.; Mertz, D.; Begin-Colin, S.; Thanh, N. T. K. Polyol synthesis, functionalisation, and biocompatibility studies of superparamagnetic iron oxide nanoparticles as potential MRI contrast agents. Nanoscale 2016, 8, 32783287.

(24) Caruntu, D.; Caruntu, G.; O'Connor, C. J. Magnetic properties of variable-sized $\mathrm{Fe}_{3} \mathrm{O}_{4}$ nanoparticles synthesized from non-aqueous homogeneous solutions of polyols. J. Phys. D: Appl. Phys. 2007, 40, 5801-5809.

(25) Jia, B.; Gao, L. Morphological transformation of $\mathrm{Fe}_{3} \mathrm{O}_{4}$ spherical aggregates from solid to hollow and their self-assembly under an external magnetic field. J. Phys. Chem. C 2008, 112, 666-671.

(26) Ge, J.; Hu, Y.; Biasini, M.; Beyermann, W. P.; Yin, Y. Superparamagnetic magnetite colloidal nanocrystal clusters. Angew. Chem., Int. Ed. 2007, 46, 4342-4345.

(27) Xu, F.; Cheng, C.; Chen, D.-X.; Gu, H. Magnetite nanocrystal clusters with ultra-high sensitivity in magnetic resonance imaging. ChemPhysChem 2012, 13, 336-341.

(28) Cullity, B. D.; Graham, C. D. Introduction to Magnetic Materials; John Wiley \& Sons, 2011.

(29) Gerischer, H. In Elektrodenpotentiale: Tables of Standard Electrode Potentials; Milazzo, H. v. G.; Caroli, S., Eds.; John Wiley \& Sons: Chichester, New York, 1978.

(30) Poul, L.; Ammar, S.; Jouini, N.; Fievet, F.; Villain, F. Synthesis of inorganic compounds (metal, oxide and hydroxide) in polyol medium: A versatile route related to the sol-gel process. J. Sol-Gel Sci. Technol. 2003, 26, 261-265.

(31) Privman, V. Diffusional nucleation of nanocrystals and their selfassembly into uniform colloids. J. Optoelectron. Adv. Mater. 2008, 10, 2827-2839.

(32) Cornell, R. M.; Schwertmann, U. The Iron Oxides: Structure, Properties, Reactions, Occurrences and Uses; John Wiley \& Sons, 2003.

(33) Barrón, V.; Torrent, J.; de Grave, E. Hydromaghemite, an intermediate in the hydrothermal transformation of 2-line ferrihydrite into hematite. Am. Mineral. 2003, 88, 1679-1688.

(34) Fock, J.; Bogart, L. K.; Gonzalez-Alonzo, D.; Martinez, J. I. E.; Hansen, M. F.; Varon, M.; Frandsen, C.; Pankhurst, Q. A. On the 'Center of Gravity' Method for Measuring the Composition of Magnetite/maghemite Mixtures, or the Stoichiometry of MagnetiteMaghemite Solid Solutions, via ${ }^{57} \mathrm{Fe}$ Mössbauer Spectroscopy. J. Phys. D: Appl. Phys., in preparation, 2017.

(35) Skrabalak, S. E.; Wiley, B. J.; Kim, M.; Formo, E. V.; Xia, Y. On the polyol synthesis of silver nanostructures: glycolaldehyde as a reducing agent. Nano Lett. 2008, 8, 2077-2081.

(36) Baronov, A.; Bufkin, K.; Shaw, D. W.; Johnson, B. L.; Patrick, D. L. A simple model of burst nucleation. Phys. Chem. Chem. Phys. 2015, 17, 20846-20852.

(37) Thanh, N. T. K.; Maclean, N.; Mahiddine, S. Mechanisms of nucleation and growth of nanoparticles in solution. Chem. Rev. 2014, $114,7610-7630$.

(38) Navrotsky, A.; Mazeina, L.; Majzlan, J. Size-driven structural and thermodynamic complexity in iron oxides. Science 2008, 319, 16351638.

(39) Serna, C. J.; Morales, M. P. Maghemite $\left(\gamma-\mathrm{Fe}_{2} \mathrm{O}_{3}\right)$ : A Versatile Magnetic Colloidal Material. Surface and Colloid Science; Springer, 2004; Vol. 17, pp 27-81. 
(40) Kostopoulou, A.; Brintakis, K.; Vasilakaki, M.; Trohidou, K. N.; Douvalis, A. P.; Lascialfari, A.; Manna, L.; Lappas, A. Assemblymediated interplay of dipolar interactions and surface spin disorder in colloidal maghemite nanoclusters. Nanoscale 2014, 6, 3764-3776.

(41) Kuchkina, N. V.; Morgan, D. G.; Kostopoulou, A.; Lappas, A.; Brintakis, K.; Boris, B. S.; Yuzik-Klimova, E. Y.; Stein, B. D.; Svergun, D. I.; Spilotros, A.; et al. Hydrophobic periphery tails of polyphenylenepyridyl dendrons control nanoparticle formation and catalytic properties. Chem. Mater. 2014, 26, 5654-5663.

(42) Liu, J.; Sun, Z.; Deng, Y.; Zou, Y.; Li, C.; Guo, X.; Xiong, L.; Gao, Y.; Li, F.; Zhao, D. Highly Water-Dispersible Biocompatible Magnetite Particles with Low Cytotoxicity Stabilized by Citrate Groups. Angew. Chem. 2009, 121, 5989-5993.

(43) Kozissnik, B.; Bohorquez, A. C.; Dobson, J.; Rinaldi, C. Magnetic fluid hyperthermia: advances, challenges, and opportunity. Int. J. Hyperthermia 2013, 29, 706-714.

(44) Vergés, M. A.; Costo, R.; Roca, A. G.; Marco, J. F.; Goya, G. F.; Serna, C. J.; Morales, M. P. Uniform and water stable magnetite nanoparticles with diameters around the monodomain-multidomain limit. J. Phys. D: Appl. Phys. 2008, 41, No. 134003.

(45) Blanco-Andujar, C.; Ortega, D.; Southern, P.; Pankhurst, Q. A.; Thanh, N. T. K. High performance multi-core iron oxide nanoparticles for magnetic hyperthermia: microwave synthesis, and the role of coreto-core interactions. Nanoscale 2015, 7, 1768-1775.

(46) Atkinson, W. J.; Brezovich, I. A.; Chakraborty, D. P. Usable frequencies in hyperthermia with thermal seeds. IEEE Trans. Biomed. Eng. 1984, BME-31, 70-75. 